Pure and Applied Mathematics Quarterly

Volume 3, Number 4

(Special Issue: In honor of

Grisha Margulis, Part 1 of 2)

1057-1099, 2007

\title{
Flat Projective Structures on Surfaces and Cubic Holomorphic Differentials
}

\author{
François Labourie \\ Pour Grisha Margulis, avec admiration
}

\section{INTRODUCTION}

The purpose of this article is to give an interpretation of real projective structures and associated cohomology classes in terms of connections, sections, etc. satisfying elliptic partial differential equations in the spirit of Hodge theory. We shall also give an application of these results as the uniqueness of a minimal surface in a symmetric space.

We recall briefly that a flat real projective structure on a surface $\Sigma$ is an atlas with values in $\mathbb{R P}^{2}$ and coordinates changes in $P S L(3, \mathbb{R})$. Associate to such a structure is a holonomy representation of $\pi_{1}(\Sigma)$ with values in $\operatorname{PSL}(3, \mathbb{R})$, and a developing map, defined from $\tilde{\Sigma}$, the universal cover of $\Sigma$, with values in $\mathbb{R} \mathbb{P}^{2}$ and equivariant under $\rho$. Finally, the structure is said to be convex if the image of the developing map is a convex set.

Convex projective structures have been extensively studied by Choi Suhyoung and William Goldman in [5] and [4] for instance. We summarise some of their major results in the following

Theorem 1.0.1. [ChOI-Goldman] Every convex structure on $\Sigma$ is determined by its holonomy representation. Moreover, if a representation of $\pi_{1}(\Sigma)$ in $P S L(3, \mathbb{R})$ can be deformed into a discrete faithful representation in $S O(2,1)$, then it is the holonomy of a convex structure on $\Sigma$.

In the introductory Section 2, we give various points of view on projective structures.

Received August 11, 2006. 
Most of the results of this paper can be stated as bijections between moduli spaces and set of solutions of certain equations. Of course the important point, not always clear in the statements, is the construction of the bijection. We now give a sketch of the content of this article. Most of the material of this article is new, although some results were announced a long time ago

Convex projective structures and cubic holomorphic differentials. Concerning convex projective structures, we prove

Theorem 1.0.2. There exists a mapping class group equivariant homeomorphism between the moduli space of convex structures on $\Sigma$ and the moduli space of pairs $(J, Q)$ where $J$ is a complex structure on $\Sigma$ and $Q$ is a cubic holomorphic differential on $\Sigma$ with respect to $J$.

This result is a combination of Theorem 4.2.1 and 4.1.1. This result was announced in [13]. The proof presented in the present paper uses a self contained approach. However, this result, as it is explained in Section 8, can be obtained as a consequence of difficult results in affine differential geometry of Cheng and Yau [2] and [3] later completed and clarified by the work of Gigena [6], Sasaki [20] and A.M. Li [17], [18]. In [19], John Loftin also proves and extends this result using this affine differential geometric interpretation.

Projective structure and cohomology classes. In Section 5, we associate to every flat projective structure of holonomy $\rho$, a nonempty cone in $H_{\rho}^{1}\left(\mathbb{R}^{3}\right)$. We also prove that this cone helps to distinguish convex structures from others. Indeed, this cone contains 0 if and only if the structure is convex.

Cohomology classes and complex structures. We obtain two results which parametrise the moduli space of representations of the surface group in the affine space in dimension 3: Theorem 6.0.10 which defines a map from $H_{\rho}^{1}(E)$ to Teichmüller space, and Theorem 6.0.11 which can be though as a generalisation of the Eichler-Shimura isomorphism in this context.

Dualities and symmetries. We show that the above results, more specially Theorem 6.0.10 and Theorem 6.0.11, give rise to unexpected symmetries of the moduli space of representations of the surface group in the affine space in dimension 3.

Higgs bundle interpretation. We also interpret Theorem 1.0.2 in the context of Higgs bundle theory. For instance, using this result, we obtain that the energy map on Teichmüller space associated to the holonomy representation of a projective structure has a unique critical point which is minimum. We note that this function is proper according to [16]. We obtain in particularly Corollary 9.3.3 that states the existence and uniqueness of a minimal surface in some symmetric spaces. 
Holomorphic interpretation. In Section 10, we explain how Theorem 1.0.2 can be interpreted as the existence and uniqueness of an equivariant "holomorphic" curve in $S L(3, \mathbb{R}) / S L(2, \mathbb{R})$.

Appendices. Finally, Appendices 13 and 12 contain compactness results for partial differentials equations appearing in the paper, arising as consequences of an holomorphic interpretations in the spirit of [12], and [14].

\section{Contents}

1. Introduction 1057

2. Projective structures 1060

2.1. First definitions. 1060

2.2. Projective structures and connections 1061

3. Convex projective structures 1064

3.1. Hypersurfaces and convex $\mathbb{R P}^{n}$-structures $\quad 1065$

3.2. Convex $\mathbb{R P}^{n}$-structures and connections 1066

4. Cubic holomorphic differentials and convex $\mathbb{R P}^{2}$ - structures. 1069

4.1. From cubic holomorphic differentials to convex $\mathbb{R P}^{2}$ - structures. 1069

4.2. From convex $\mathbb{R P}^{2}$-structures to cubic holomorphic differentials 1073

$\begin{array}{lll}\text { 5. Projective structures and cohomology classes } & 1077\end{array}$

6. Cohomology classes and complex structures 1079

6.1. Cohomology classes and complex structures 1080

6.2. Hodge representatives 1083

7. Dualities and symmetries of moduli spaces 1084

7.1. Contragredient representation and Theorem 4.2.1 1084

7.2. Theorem 6.0.10 and an involution on the moduli space of representations in the affine group $\quad 1085$

7.3. Theorem 6.0.11 and a fourth order symmetry on the moduli space of representations in the affine group $\quad 1085$

8. An affine differential interpretation 1086

8.1. Hyperbolic affine spheres and Theorem 4.2.1 1087

8.2. Constant Gaussian curvature surfaces and Theorem 6.0.10 1087 
8.3. $\quad$ Interpretation of Theorem 5.0.8 1088

9. A Higgs bundle interpretation 1088

9.1. Representations and harmonic mappings 1088

9.2. Representations, energy and minimal surfaces 1089

9.3. The case of $n=3$. 1090

10. A holomorphic interpretation 1092

11. Appendix A: geodesics 1092

12. Appendix B: Elliptic Monge-Ampère equations. 1093

13. Appendix C: Laplace equations 1095

14. Appendix D: the Böchner formula 1097

\section{Projective structures}

2.1. First definitions. Let $M$ be a manifold of dimension $n$.

2.1.1. Projectively equivalent connections. On a manifold $M$ two connections are said to be projectively equivalent if they have the same geodesics, up to parametrisations. In dimension greater than 2 , two torsion free connections $\nabla^{1}$ and $\nabla^{2}$ are projectively equivalent if there exist a 1 -form $\beta$ such that

$$
\nabla_{X}^{1} Y-\nabla_{X}^{2} Y=\beta(X) Y+\beta(Y) X .
$$

A class of projectively equivalent connections defines a projective structure on $M$. Projective structures can be induced by local diffeomorphisms.

2.1.2. Projectively flat structures. A projective structure is flat if every point has a neighbourhood on which the projective structure is given by a torsion free flat connection.

The projective space $\mathbb{R P}^{n}$ admits a projectively flat structure given by the affine charts. Conversely, a manifold $M$ of dimension $n$ is equipped with a flat projective structure, if there exist

- a representation $\rho$-the holonomy representation- of $\pi_{1}(M)$, the fundamental group of $M$, with values in the projective group $P S L(n,+1, \mathbb{R})$;

- a local diffeomorphism $f$, the developing map, of the universal cover $\widetilde{M}$ of $M$ with values in $\mathbb{R P}^{n}$, which is $\rho$-equivariant, that is which satisfies

$$
\forall x \in \widetilde{M}, \forall \gamma \in \pi_{1}(M), \quad f(\gamma x)=\rho(\gamma) f(x) .
$$


The structure on $M$ is the one induced by the projective structure on $\mathbb{R} \mathbb{P}^{n}$ by $(f, \rho)$.

In other words, a flat projective structure on a manifold is nothing else that a $\left(\mathbb{R} \mathbb{P}^{n}, P S L(n,+1, \mathbb{R})\right)$-structure, or, in short, an $\mathbb{R} \mathbb{P}^{n}$-structure. We shall restrict ourselves in this article to structures such that the holonomy representation lifts to $\operatorname{PSL}(n, \mathbb{R})$.

2.1.3. Convex structures. A $\mathbb{R} \mathbb{P}^{n}$-structure is convex if the developing map is a homeomorphism to a convex set in $\mathbb{R} \mathbb{P}^{n}$. It is properly convex if this convex set is included in a compact convex set of an affine chart.

2.2. Projective structures and connections. We explain and relate in this section two different points of view on projectively flat structures.

- In the first paragraph, we explain that pairs consisting of a flat connection and a section of a rank $n+1$ vector bundle over an $n$-dimensional manifold can give rise to flat projective structures.

- In the second paragraph, we explain that the pair consisting of a torsion free connection and a symmetric tensor - satisfying some compatibility condition - on a manifold also gives to a flat structure

2.2.1. Flat connections and sections. We consider a bundle $E_{M}$ of rank $n+1$ over $M$ equipped with a volume form $\omega$. Let $\nabla$ be a connection on $E_{M}$ preserving the volume form. We observe that each section $u$ of $E_{M}$, defines a section $\Omega_{u}^{0}$ of $\Lambda^{n}\left(T M^{*}\right)$, given by

$$
\Omega_{u}^{0}\left(X_{1}, \ldots, X_{n}\right)=\omega\left(\nabla_{X_{1}} u, \ldots, \nabla_{X_{n}} u, u\right) .
$$

We say $u$ is $\nabla$-immersed if $\Omega_{u}^{0}$ is non degenerate.

We now relate these notions to projective structures. If $\nabla$ is flat, we identify the lift $E_{\widetilde{M}}$ of $E_{M}$ on the universal cover $\widetilde{M}$ of $M$ with $E \times \widetilde{M}$ so that $\nabla$ is the trivial connection. Let $\rho$ be the holonomy representation of $\nabla$. A section $u$ of $E_{M}$ is then identified with a $\rho$-equivariant map from $\widetilde{M}$ to $E$. We denote by $x \rightarrow[x]$ the projection from $E \backslash\{0\}$ to $\mathbb{P}(E)$. A section $u$ is $\nabla$-immersed, if it is a nonzero section and if the associated $\rho$-equivariant map $[u]$ from $\widetilde{M}$ to $\mathbb{P}(E)$ is an immersion.

It follows that a pair $(\nabla, u)$ such that $\nabla$ is flat and $u$ is $\nabla$-immersed gives rise to a flat projective structure. Conversely it is immediate to check every flat projective structure whose holonomy lifts to $\operatorname{PSL}(n,+1, \mathbb{R})$ is obtained this way, maybe after going to a double cover.

We obtain immediately from the previous construction the following proposition 
Proposition 2.2.1. If $\left(\nabla_{1}, u_{1}\right)$ and $\left(\nabla_{2}, u_{2}\right)$ give rise to two flat projective structures equivalent by a diffeomorphism $\phi$, then there exist

- a bundle automorphism $\Phi$ of $E_{M}$ over $\phi$,

- a nonzero function $f$,

such that $\Phi^{*} \nabla_{1}=\nabla_{2}$ and $\Phi^{*} u_{1}=f u_{2}$.

2.2.2. Connections and symmetric tensors. Let $\nabla$ be a torsion free connection on $M$. Let $h$ be a symmetric two-tensor on $M$. Let $L=\mathbb{R} \times M$ be the trivial real vector bundle of rank one over $M$. We associate to the pair $(\nabla, h)$ a connection $\nabla^{h}$ on $T M \oplus L$ given by

$$
\nabla_{X}^{h}\left(\begin{array}{c}
Z \\
\lambda
\end{array}\right)=\left(\begin{array}{c}
\nabla_{X} Z+\lambda X \\
L_{X} \lambda+h(Z, X)
\end{array}\right)
$$

We say $(\nabla, h)$ satisfy Condition $(E)$ if,

- $\nabla$ preserves a volume form,

- $\nabla^{h}$ is flat.

Note that if $\nabla$ satisfies Condition (E), then $\nabla^{h}$ preserves a volume form on $T M \oplus L$. Moreover, the conditions that $\nabla$ is torsion free and $h$ symmetric are redundant. Finally, $\nabla^{h}$ is flat if and only if the following two equations are satisfied.

$$
\left\{\begin{array}{r}
d^{\nabla} h(X, Y, Z)=\nabla_{X} h(Y, Z)-\nabla_{Y} h(X, Z)=0 \\
R^{\nabla}(X, Y) Z-h(X, Z) Y+h(Y, Z) X=0
\end{array}\right.
$$

We now relate this to the previous paragraph. Let as above $\bar{\nabla}$ be a connection on $E_{M}$ preserving a volume form $\omega$. We observe that each section $u$ of $E_{M}$, defines a section $\Omega_{u}^{2}$ of $\Lambda^{n}\left(T M^{*}\right) \otimes S^{2}(T M)$, by the following formula

$$
\Omega_{u}^{2}\left(X_{1}, \ldots, X_{n}, Y, Z\right)=\omega\left(\bar{\nabla}_{X_{1}} u, \ldots, \bar{\nabla}_{X_{n}} u, \bar{\nabla}_{Z} \bar{\nabla}_{Y} u\right) .
$$

If $u$ is $\bar{\nabla}$-immersed, then we can write

$$
\Omega_{u}^{2}=\Omega_{u}^{0} \otimes S_{u},
$$

where $S_{u}$ is a section of $S^{2}(T M)$. Note that the symmetric two tensor $S_{u}=S_{u}^{\bar{\nabla}}$ is independent of the choice of $\omega$.

Let $u_{0}$ is the canonical section of $T M \oplus L$, defined by

$$
u_{0}=(0,1) \text {. }
$$

Then $h=S_{u_{0}}^{\nabla^{h}}$. Conversely if $\left(\nabla^{0}, u\right)$ is a pair such that $u$ is $\nabla$-immersed, then the following mapping is an isomorphism

$$
\Phi:\left\{\begin{aligned}
T M \oplus L & \rightarrow E_{M} \\
(X, \lambda) & \mapsto \nabla_{X} u+\lambda u
\end{aligned}\right.
$$


Moreover there exists a connection $\nabla=\nabla^{u}$ on $M$ such that

$$
\Phi^{*} \nabla^{0}=\nabla^{h}, \text { with } h=S_{u}^{\nabla^{0}} .
$$

This construction is related to projective structures by the following result.

Proposition 2.2.2. If $(\nabla, h)$ satisfies Condition $(E)$, then $\nabla$ is projectively flat and the holonomy of the corresponding structure is the holonomy representation of $\nabla^{h}$. Conversely, every flat projective structure on $M$, whose holonomy lifts to $\operatorname{PSL}(n,+1, \mathbb{R})$ is obtained this way.

Proof : the only point to be proved is that $\nabla$ is projectively flat and defines the same projective structure. Let $\gamma(t)$ be a geodesic for $\nabla$, then the sub-bundle

$$
P=\mathbb{R} \dot{\gamma}(t) \oplus \mathbb{R} \subset T M \oplus L \mathbb{R},
$$

is parallel along $\gamma(t)$. Therefore, using the local trivialisation given by $\nabla^{h},[u(\gamma(t)]$ is the projective line defined by $P$. Q.E.D.

We also prove an independent - and technical - proposition which is going to be useful in the sequel.

Proposition 2.2.3. Let $E_{M}$ be a bundle of rank $n+1$ over $M$ equipped with a connection $\nabla$. Let $u$ be a $\nabla$-immersed section of $E_{M}$. Then there exists a connection $\nabla^{u}$ on $T M$ such that

$$
\nabla_{X} \nabla_{Y} u=S_{u}(X, Y) \cdot u+\nabla_{\nabla_{X}^{u} Y} u
$$

Moreover

$$
\begin{aligned}
S_{\frac{u}{f}} & =S_{u}-\frac{\left(\nabla^{u}\right)^{2} f}{f}, \\
\nabla_{X}^{\frac{u}{f}} Y & =\nabla_{X}^{u} Y-\frac{d f(X)}{f} Y-\frac{d f(Y)}{f} X .
\end{aligned}
$$

Proof : We consider the linear operator $\Pi_{u}: E_{M} \rightarrow T M$, such that

$$
\Pi_{u}(u)=0, \quad \Pi_{u}\left(\nabla_{X} u\right)=X .
$$

We now observe that the operator $\nabla^{u}: \Gamma(T M)^{2} \rightarrow \Gamma(T M)$ defined by

$$
\nabla_{X}^{u} Y=\Pi_{u}\left(\nabla_{X} \nabla_{Y} u\right),
$$

is actually a connection. By construction

$$
\nabla_{X} \nabla_{Y} u=S_{u}(X, Y) \cdot u+\nabla_{\nabla_{X}^{u} Y} u .
$$

Next, we consider $\pi_{u}: E_{M} \rightarrow \mathbb{R}$, such that

$$
\pi_{u}(u)=0, \quad \pi_{u}\left(\nabla_{X} u\right)=0 .
$$


Then

$$
0=\pi_{\frac{u}{f}}\left(\nabla_{X}\left(\frac{u}{f}\right)\right)=\frac{1}{f}\left(\pi_{\frac{u}{f}}\left(\nabla_{X} u\right)-d f(X)\right) .
$$

Thus,

$$
\pi_{\frac{u}{f}}\left(\nabla_{X} u\right)=d f(X)
$$

Also

$$
\begin{aligned}
\nabla_{X} \nabla_{Y} \frac{u}{f} & =\frac{1}{f} \nabla_{X} \nabla_{Y} u-\frac{1}{f^{2}} L_{X} L_{Y} f u \\
& +2 \frac{d f(X) d f(Y)}{f^{3}} u-\frac{d f(X)}{f^{2}} \nabla_{Y} u--\frac{d f(Y)}{f^{2}} \nabla_{X} u
\end{aligned}
$$

By definition, $S_{u}(X, Y)=\pi_{u}\left(\nabla_{X} \nabla_{Y} u\right)$. Then

$$
\begin{aligned}
S_{\frac{u}{f}}(X, Y) & =\pi_{\frac{u}{f}}\left(\nabla_{X} \nabla_{Y} \frac{u}{f}\right) \\
& \left.=-\frac{1}{f} L_{X} L_{Y} f+\frac{1}{f} \pi_{\frac{u}{f}}\left(\nabla_{X} \nabla_{Y} u\right) \cdot u\right) \\
& =-\frac{1}{f} L_{X} L_{Y} f+\frac{1}{f} \pi_{\frac{u}{f}}\left(\nabla_{\nabla_{X}^{u}}^{u} u\right)+S_{u}(X, Y) \\
& =-\frac{1}{f}\left(L_{X} L_{Y} f-d f\left(\nabla_{X}^{u} Y\right)\right)+S_{u}(X, Y) \\
& =-\frac{1}{f}\left(\nabla^{u}\right)_{X, Y}^{2} f+S_{u}(X, Y) .
\end{aligned}
$$

Finally, writing $v=\frac{u}{f}$, we have

$$
\begin{aligned}
\nabla_{\nabla_{X}^{v} Y \frac{u}{f}} & =\nabla_{X} \nabla_{Y} \frac{u}{f}-T_{v}(X, Y) \frac{u}{f} \\
& =-\frac{d f(X)}{f} \nabla_{Y} \frac{u}{f}-\frac{d f(Y)}{f} \nabla_{X} \frac{u}{f}+\nabla_{\nabla_{X}^{u} Y} \frac{u}{f}
\end{aligned}
$$

It follows that

$$
\nabla_{X}^{\frac{u}{f}} Y=\nabla_{X}^{u} Y-\frac{d f(X)}{f} Y-\frac{d f(Y)}{f} X
$$

Q.E.D.

\section{Convex projective Structures}

In this section, we explain how convex projective structures can be interpreted using the point of view of the previous Section. Our main result is Theorem 3.2.1. 
3.1. Hypersurfaces and convex $\mathbb{R}^{n}$-structures. Let $E$ be a vector space of dimension $n$. We say an immersed hypersurface $\Sigma$ in $E \backslash\{0\}$

- is locally convex, if every point in $\Sigma$ has a neighbourhood $U$ in $\Sigma$ which is a subset of the boundary of a convex set.

- is locally strictly convex, if furthermore $U$ does not contain any segment. In particular a locally strictly convex separates $E$ locally in two connected components, the interior -which coincides with the convex set - and an exterior

- We say a strictly locally convex hypersurface is radial, if the radial vector - the vector pointing from the origin - points inward.

- Finally, we observe that if $\Sigma$ is strictly locally convex, radial and properly immersed, it bounds a convex set which does not contain the origin.

Every such hypersurface admits a natural properly convex structure. Indeed the projection from $\Sigma$ to $\mathbb{P}(E)$ is an immersion. Since $\Sigma$ is strictly convex, its projection is a convex set whose closure is compact and included in an affine chart.

Conversely, the following lemma, basically due to Vinberg [23], shows every properly convex structure is obtained this way.

Lemma 3.1.1. Let $M$ be manifold equipped with a properly convex projective structure given by the pair $(f, \rho)$. Then there exists a proper $\rho$-equivariant immersion $g$ of $\widetilde{M}$ whose image is strictly convex and radial and such that $\pi \circ g=f$ where $\pi$ is the projection $E \backslash\{0\}$ on $\mathbb{P}(E)$.

Proof : Let $\mathcal{C}$ be an open proper convex set of $\mathbb{P}(E)$. Let $C^{0}$ be the cone of $E$ obtained from $\mathcal{C}$

$$
C^{0}=\pi^{-1}(\mathcal{C}) .
$$

Let $C$ be the convex cone which is one the two connected component of $C^{0}$. Let $C^{*}$ be the dual cone of $C$. Let $d f$ be a volume form on the dual $E^{*}$ of $E$. Let $V$ be the characteristic function of Vinberg [23] defined by

$$
\forall x \in C, V(x)=\int_{C^{*}} e^{-f(x)} d f .
$$

This function $V$ is convex and one shows easily that the hypersurface $\Sigma=V^{-1}\{1\}$ is properly immersed strictly convex and radial and $\pi$ is a diffeomorphism from $\Sigma$ to $\mathcal{C}$.

Since $V$ is invariant by the subgroup of the special affine group that leaves $C$ invariant, we obtain that $\Sigma$ is also invariant. 
In particular, if $f$ is the developing map of a properly convex structure. We can define

$$
g:\left\{\begin{aligned}
\widetilde{M} & \rightarrow \Sigma \\
x & \mapsto \pi^{-1}(f(x)) .
\end{aligned}\right.
$$

The mapping $g$ satisfies the condition of the lemma. Q.E.D.

3.2. Convex $\mathbb{R} \mathbb{P}^{n}$-structures and connections. We explain in this section that a properly convex $\mathbb{R} \mathbb{P}^{n}$-structure on a compact manifold $M$ is equivalent to the data of a connection $\nabla$ on $T M$, and a positive definite metric $g$ on $T M$ such that $(\nabla, g)$ satisfies Condition (E). We use the language of Paragraph 2.2.2

Theorem 3.2.1. If $(\nabla, g)$ satisfies Condition (E) and $g$ is positive definite, then $\nabla$ is projectively flat and defines a properly convex structure on $M$. Conversely, every properly convex projectively flat structure on $M$ is obtained this way.

The referee observed that an analogous result - for locally flat manifolds - was proved by J.-L Koszul in [10].

Let $\widetilde{M}$ be the universal cover of $M$. By a slight abuse of notation, we shall also denote by $L$ the trivial rank 1 vector bundle over $\widetilde{M}$. We first observe that since $\nabla^{g}$ is flat, the bundle $T \widetilde{M} \oplus L$ over $\widetilde{M}$ is isomorphic to the trivial bundle $E \times \widetilde{M}$, where $E$ is an $n+1$-dimensional.

- Let $p$ be the projection from $T \widetilde{M} \oplus L$ to $E$,

- Let $\rho$ be the holonomy representation of the flat connection $\nabla^{g}$,

- Let $u_{0}: m \rightarrow(0,1)$ be the canonical section of $T \widetilde{M} \oplus L$ - where $0 \in T_{m} \widetilde{M}$ and $1 \in \mathbb{R}$.

- Let $\phi=p \circ u_{0}$.

By construction, $\phi$ is a $\rho$-equivariant mapping from $\widetilde{M}$ to $E$.

We prove the Theorem in several steps

Proposition 3.2.2. The mapping $\phi$ is an immersion.

Proof : We have by construction

$$
D \phi(X)=p\left(\nabla_{X}^{g} u_{0}\right)=p(X, 0) .
$$

It follows $\phi$ is an immersion. Q.E.D.

Proposition 3.2.3. The immersed hypersurface $\phi(\widetilde{M})$ is strictly locally convex and radial.

Proof : Let $\Sigma$ be a hypersurface in $E$. For every $s \in \Sigma$, let $\pi_{s}$ be the projection $E \rightarrow E / T_{s} \Sigma$. Let $D$ be the flat connection in $E$. For every vector field 
$X$ on $\Sigma$, we recall that there exists a symmetric 2 -tensor $B$ such that

$$
\pi_{s}\left(D_{X} X\right)=B\left(X_{s}, X_{s}\right)
$$

A hypersurface is strictly convex at $s$ if and only if for every non zero vector $X$ in $T_{s} \Sigma$, we have

$$
B\left(X_{s}, X_{s}\right) \neq 0
$$

In our case, let $\Sigma=\phi(\widetilde{M})$, then

$$
B\left(X_{s}, X_{s}\right)=g\left(X_{s}, X_{s}\right)>0 .
$$

This also prove that $\Sigma$ is radial. Q.E.D.

The next proposition is less straightforward.

Proposition 3.2.4. $\phi$ is proper.

Proof : We first note that the geodesics for $\nabla$ are precisely those curves whose image by $\phi$ lies on 2-plane which passes through the origin. Now

- Let $x_{0}$ a point in $\widetilde{M}$,

- Let $U$ the domain of $T_{x_{0}} \widetilde{M}$ on which the exponential map exp for $\nabla$ is defined,

- Let $v$ be a nonzero vector in $T_{x_{0}} \widetilde{M}$,

- Let $I=] a, b[$ be the maximal interval for which $I v \subset U$,

- Let $c_{0}(t)=\exp (t v)$.

We first prove that the map $c: I \rightarrow E$ - depending on $v$-defined by

$$
c(t)=\phi\left(c_{0}(t)\right)
$$

is proper. We know that $c$ is a strictly convex planar curve which is furthermore radial. To prove that $c$ is proper it suffices to show that the length of this curve is infinite for an euclidean metric $\langle$,$\rangle on E$. Let $\mu(t)=\sqrt{g\left(\dot{c_{0}}(t), \dot{c_{0}}(t)\right)}$. By definition,

$$
\nabla_{\dot{c}(t)}^{g} \dot{c_{0}}(t)=g\left(\dot{c_{0}}(t), \dot{c_{0}}(t)\right) \cdot u_{0}\left(c_{0}(t)\right)
$$

Hence,

$$
\ddot{c}=p\left(\nabla_{\dot{c}_{0}(t)}^{g} \dot{c_{0}}(t)\right)=g\left(\dot{c_{0}}(t), \dot{c_{0}}(t)\right) \cdot p \circ u_{0}\left(c_{0}(t)\right)=\mu^{2} c
$$

Let $\lambda(t)=\sqrt{\langle\dot{c}(t), \dot{c}(t)\rangle}$. We have

$$
\begin{aligned}
\frac{d \lambda}{d t} & =\frac{\langle\dot{c}, \ddot{c}\rangle}{\lambda} \\
& =\mu^{2} \cdot \frac{\langle\dot{c}, c\rangle}{\lambda}
\end{aligned}
$$


We now prove that $t \rightarrow \frac{\langle\dot{c}(t), c(t)\rangle}{\lambda(t)}$ is increasing:

$$
\begin{aligned}
\frac{d}{d t} \frac{\langle\dot{c}, c\rangle}{\lambda} & =\frac{\lambda(\langle\ddot{c}, c\rangle+\langle\dot{c}, \dot{c}\rangle)-\mu^{2} \lambda^{-1}\langle c, \dot{c}\rangle^{2}}{\lambda^{2}} \\
& =\frac{\mu^{2} \lambda\langle c, c\rangle+\lambda\langle\dot{c}, \dot{c}\rangle-\mu^{2} \lambda^{-1}\langle c, \dot{c}\rangle^{2}}{\lambda^{2}} \\
& =\frac{\left(\langle c, c\rangle\langle\dot{c}, \dot{c}\rangle-\langle c, \dot{c}\rangle^{2}\right) \mu^{2} \lambda^{-1}}{\lambda^{2}}+\frac{\langle\dot{c}, \dot{c}\rangle}{\lambda} \\
& \geq 0 .
\end{aligned}
$$

Let us choose the euclidean metric such that $\langle\dot{c}(0), c\rangle>0$. Thus, there exists $K_{1}>0$ such that

$$
\forall t>0, \frac{\langle\dot{c}(t), c\rangle}{\lambda(t)} \geq K_{1}
$$

Hence, by Formula (5),

$$
\forall t>0, \quad \frac{d \lambda}{d t} \geq K_{1} \mu^{2} .
$$

But, by Lemma 11.0.5 of the Appendix.

$$
\forall t,\left|\frac{d \mu}{d t}\right| \leq K_{2} \mu^{2}
$$

Thus

$$
\forall t>0, \quad \frac{d \lambda}{d t} \geq K_{3} \frac{d \mu}{d t} .
$$

It follows again by Lemma 11.0.5 of the Appendix that

$$
\int_{0}^{b} \lambda(s) d s \geq K_{3} \int_{0}^{b} \mu(s) d s+K_{4}=+\infty .
$$

Finally, we show that $\phi \circ \exp$ is proper. Let $\left\{u_{n}\right\}_{n \in \mathbb{N}}$ be a sequence of points in $U$ such that $\left\{\phi \circ \exp \left(u_{n}\right)\right\}_{n \in \mathbb{N}}$ converge to $z_{0}$. By choosing a subsequence, we may suppose that the sequence of lines $\left\{D_{n}=\mathbb{R} u_{n}\right\}_{n \in \mathbb{N}}$ converges to $D_{0}$, and that according to the previous discussion $-\left\{\phi \circ \exp \left(D_{n} \cap U\right)\right\}_{n \in \mathbb{N}}$ converges to a locally convex radial curve $c$. But then, $\phi \circ \exp \left(D_{0} \cap U\right)$ is an open set in $c$, and since it has infinite length it coincides with $c$. Thus, $\left\{u_{n}\right\}_{n \in \mathbb{N}}$ converges to a point $u_{0}$ in $D_{0} \cap U$ such that $\phi \circ \exp \left(u_{0}\right)=z_{0}$. Q.E.D.

3.2.1. Proof of Theorem 3.2.1. We prove the first part of the Theorem. By Proposition 3.2.3, 3.2.2 and 3.2.4, $\phi$ is a proper immersion and $\phi(M)$ is a locally convex proper hypersurface. By Section 3.1, we can construct a flat projective structure on $M$, whose projective lines are the intersection of 2-planes with $\phi(M)$. But the latter are geodesics on $\widetilde{M}$ for $\nabla$, therefore $\nabla$ is projectively flat. 
Conversely, by Lemma 3.1.1, any properly convex projectively flat structure on $M$ can be induced by proper $\rho$-equivariant immersion in $E$ of $\widetilde{M}$ whose image is a locally strictly convex hypersurface $\Sigma$. Since $\Sigma$ is radial, we have the decomposition

$$
\left.T E\right|_{\Sigma}=T \Sigma \oplus \mathbb{R} R
$$

The flat connexion $\nabla^{0}$ on $\left.T E\right|_{\Sigma}$ decomposes therefore, if $Z$ is a vector field on $\Sigma$ as

$$
\nabla_{X}^{0}(Z+\lambda R)=\nabla_{X} Z+\lambda \nabla_{X}^{0} R+L_{X} \lambda \cdot R+g(Z, X) \cdot R .
$$

We observe that $\nabla_{X}^{0} R=X$, and $g(X, X)>0$ since $\Sigma$ is strictly locally convex and radial. It follows that $(\nabla, g)$ satisfies Condition (E) 2 .

\section{Cubic holomorphic differentials And CONVEx $\mathbb{R P}^{2}$ - StRUCtures.}

The two main results of this section, Theorem 4.1.1 and 4.2.1, provide a bijection of the space of pairs consisting of a complex structure and a cubic holomorphic differential, with the moduli space of convex projective structures.

\subsection{From cubic holomorphic differentials to convex $\mathbb{R P}^{2}$ - structures.}

The content of this section is another version of Wang Chang Pin result [24] which states that every cubic holomorphic differential on a compact surface gives rise to an affine sphere. The proofs are slightly different. However for the sake of completeness, and to make this article as self contained as possible, we recall the construction.

First, we introduce notations

- If $\nabla^{0}$ is a connection on $T \Sigma$ and $B$ is a section of $T \Sigma^{*} \otimes \operatorname{End}(T \Sigma)$, we denote by $\nabla^{0}+B$ the connection on $T \Sigma$ defined by

$$
\left(\nabla^{0}+B\right)_{X} Y=\nabla_{X}^{0} Y+B(X) Y .
$$

- Conversely, if $\nabla$ and $\nabla^{0}$ are two connections on $T \Sigma$, then $\nabla-\nabla^{0}$ will denote the section of $T \Sigma^{*} \otimes \operatorname{End}(T \Sigma)$ defined by

$$
\left(\nabla-\nabla^{0}\right)(X) Y=\nabla_{X} Y-\nabla_{X}^{0} Y .
$$

Theorem 4.1.1. Let $\omega$ be a cubic holomorphic differential on a Riemann surface $(\Sigma, J)$. For any metric $g$, let $A_{g}$ be the section of $T \Sigma^{*} \otimes \operatorname{End}(T \Sigma)$ defined by

$$
g\left(A_{g}(X) Y, Z\right)=\Re(\omega(X, Y, Z)) .
$$

Then there exists a unique metric $g$ in the conformal class of $J$ such that if $\nabla$ is the Levi-Civita connexion of $g$, then $\left(\nabla+A_{g}, g\right)$ satisfies Condition $(E)$. Moreover the area for $g$ is parallel for $\nabla+A_{g}$. 
We explain how to reduce the theorem to the existence and uniqueness of a solution of an elliptic differential equation proved in Lemma 4.1.2.

Let $g$ be a metric conformal to $J$. Let $\nabla$ be the Levi-Civita connection of $g$. Let $A$ be a section of $T \Sigma^{*} \otimes \operatorname{End}(T \Sigma)$. Then a straightforward check shows that $(\nabla+A, g)$ satisfies Condition $(E)$ if and only if

(1) $A(X)$ is symmetric and trace free

(2) $A(X) Y=A(Y) X$

(3) $d^{\nabla} A=0$

(4) $R^{g}(X, Y) Z+[A(X), A(Y)] Z+g(Y, Z) X-g(X, Z) Y=0$.

Let $\omega$ be a cubic holomorphic differential on $(\Sigma, J)$ and $g$ be a metric in the conformal class of $J$. Let now $A=A_{g}$. The first three conditions are satisfied by $A$ since $\omega$ is a cubic holomorphic differential. We concentrate on the last condition. We first rewrite it in a more compact way. There exist a positive definite quadratic form $\mathcal{G}^{g}$, depending on $g$ and defined on the space of cubic differentials such that

$$
[A(X), A(Y)] Z=-\mathcal{G}^{g}(\omega, \omega)(g(Y, Z) X-g(X, Z) Y) .
$$

Note that

$$
\mathcal{G}^{\lambda g}=\frac{1}{\lambda^{3}} \mathcal{G}^{g}
$$

We can rewrite (4) as

$$
k_{g}+1-\mathcal{G}^{g}(\omega, \omega)=0
$$

where $k_{g}$ is the curvature of $g$.

Let $g_{0}$ be the metric of constant curvature -1 on $\Sigma$ in the conformal class of $J$. Let $\nabla_{0}$ be its Levi-Civita connection. We define the Laplacian with respect to $g_{0}$ by

$$
\Delta f=-\operatorname{trace}\left(\nabla_{0}^{2} f\right),
$$

where $g_{0}\left(\nabla^{2} f(u), v\right)=\left(\nabla_{0}\right)_{u} d f(v)$. In other words we have,

$$
d(d f \circ J)=\Delta f \cdot \Omega_{g_{0}},
$$

where $J$ and $\Omega_{g_{0}}$ are respectively the complex structure and volume form of $g_{0}$.

Write now $g=\lambda g_{0}$. Let $f=\mathcal{G}^{g_{0}}(\omega, \omega)$ and $\mu=\frac{1}{2} \log (\lambda)$. Recall that

$$
k_{g}=e^{-2 \mu}(\Delta \mu-1) \text {. }
$$

Then we rewrite (6) as

$$
f-e^{4 \mu} \Delta \mu-e^{6 \mu}+e^{4 \mu}=0 .
$$

Therefore the result follows from 
Lemma 4.1.2. Let $\Sigma$ be a compact hyperbolic surface. Let $f$ be a positive function on $\Sigma$. Then there exists a unique function $\mu$ such that

$$
e^{4 \mu} \Delta \mu+e^{6 \mu}-e^{4 \mu}=f
$$

where $\Delta=-\operatorname{trace}\left(\nabla^{2}\right)$ is the Laplacian.

The rest of this paragraph is devoted to the proof of this Lemma. Let

$$
H(\mu)=e^{4 \mu} \Delta \mu+e^{6 \mu}-e^{4 \mu}
$$

4.1.1. A-priori estimates. Let $\mu$ be a $C^{\infty}$-function on $\Sigma$. Let $f=H(\mu)$. We assume that $f \geq 0$. We want to control $\mu$ using $f$. Our main result is the following

Lemma 4.1.3. For every $A$, there exists $B$ such that $\|f\|_{C^{1}} \leq B$ implies $\|\mu\|_{C^{1}} \leq$ A

Proof : We first obtain $C^{0}$ estimates using the maximum principle. At a minimum of $\mu$, we have $\Delta \mu \leq 0$. Thus $f \leq e^{6 \mu}-e^{4 \mu}$. Therefore $e^{6 \mu}-e^{4 \mu} \geq 0$. Hence $\mu \geq 0$. Now at a maximum of $\mu$, we have $\Delta \mu \geq 0$ thus $f \geq e^{6 \mu}-e^{4 \mu}$. This proves that for every $A$, there exists $B$ such that $\|f\|_{C^{0}} \leq B$ implies $\|\mu\|_{C^{0}} \leq A$.

Next we prove the $C^{1}$ estimates. Since $g_{0}$ has constant curvature -1, we have the following formula

$$
\Delta\|d \mu\|^{2}=2\langle d(\Delta \mu), d \mu\rangle-2\left\|\nabla^{2} \mu\right\|^{2}+2\|d \mu\|^{2} .
$$

We give the proof of this classical formula in the Appendix D (14). Let $f=H(\mu)$, from Formula (8) one gets:

$$
\begin{aligned}
\Delta\|d \mu\|^{2} & =-\|d \mu\|^{2}\left(8 e^{-4 \mu} f+4 e^{2 \mu}-2\right)-\left\|\nabla^{2} \mu\right\|^{2}+2 e^{-4 \mu}\langle d f, d \mu\rangle \\
& \leq\left(\|d f\| \cdot\|d \mu\|-\|d \mu\|^{2}\left(4 f+2 e^{6 \mu}-e^{4 \mu}\right)\right) 2 e^{-4 \mu} .
\end{aligned}
$$

We have shown that $e^{6 \mu}-e^{4 \mu} \geq 0$ and we have assumed that $f \geq 0$. Thus

$$
4 f+2 e^{6 \mu}-e^{4 \mu}>1 \text {. }
$$

Therefore when $\|d \mu\|$ is maximum then

$$
0 \leq \Delta\|d \mu\|^{2} \leq\left(\|d \mu\|^{2}-\|d f\| \cdot\|d \mu\|\right) 2 e^{-4 \mu} .
$$

Thus

$$
\|d \mu\| \leq \sup \|d f\| .
$$

Q.E.D. 
4.1.2. Proof of Lemma 4.1.2. Let

$$
\mathcal{F}=\{\mu / H(\mu) \geq 0\}
$$

We first want to prove that

$$
H: \mathcal{F} \rightarrow C^{\infty}(\Sigma,[0,+\infty[)
$$

is a homeomorphism. Since $C^{\infty}(\Sigma,[0,+\infty[)$ is contractible and in particular simply connected, it suffices to show that

(1) $H$ is a local homeomorphism,

(2) $H$ is proper,

(3) $\mathcal{F}$ is connected.

Indeed Properties 1 and 2, implies that $H$ is a finite covering.

We prove (1). Since $H$ is an elliptic operator, it suffices to show by the local inversion theorem that the linearised operator $L_{\mu}^{H}$ of $H$ at $\mu$ is invertible. A straightforward computation yields

$$
L_{\mu}^{H}(\lambda)=4 \lambda H(\mu)+2 \lambda e^{6 \mu}+e^{4 \mu} \Delta \lambda .
$$

By hypothesis $G=4 H(\mu)+2 e^{6 \mu}>0$. We deduce that the kernel $L_{\mu}^{H}$ is reduced to zero. Indeed if $L_{\mu}^{H}(\lambda)=0$ then

$$
\begin{aligned}
0 & =\int_{\Sigma} \lambda \cdot L_{\mu}^{H}(\lambda) \\
& =\int_{\Sigma} \lambda \cdot \Delta \lambda+\int_{\Sigma} G \lambda^{2} \\
& =\int_{\Sigma}\|d \lambda\|^{2}+\int_{\Sigma} G \lambda^{2} .
\end{aligned}
$$

Finally $L_{\mu}^{H}$ is an elliptic homotopic to the Laplacian, the index of $L_{\mu}^{H}$ is zero. It follows $L_{\mu}^{H}$ is invertible

We prove (2). Assume that $\left\{H\left(\mu_{n}\right)\right\}_{n \in \mathbb{N}}$ converges. It follows from Lemma 4.1.3, that $\left\{\left\|\mu_{n}\right\|_{C^{1}}\right\}_{n \in \mathbb{N}}$ is bounded. Then classical arguments [7] shows that $\left\{\mu_{n}\right\}_{n \in \mathbb{N}}$ converges . For the sake of completeness, we give a slightly nonstandard proof of that fact in Appendix 13.

We prove (3). For the moment we have proved that $H$ is a finite index covering. To prove that $\mathcal{F}$ is connected, it suffices to show that for some $f$, there is a unique solution of $H(\mu)=f$. Indeed, for $f=0$, the equation $H(\mu)=0$ says that $e^{2 \mu} g_{0}$ has constant curvature -1 . Hence $\mu=0$. Q.E.D. 
4.2. From convex $\mathbb{R P}^{2}$-structures to cubic holomorphic differentials. We prove the following theorem

Theorem 4.2.1. Let $\Sigma$ be equipped with a convex projective structure. Then there exists a pair $(\nabla, g)$ unique up to diffeomorphism, such that

- $\nabla$ defines the projective structure,

- $g$ is positive definite,

- $\nabla \omega_{g}=0$, where $\omega_{g}$ is the volume form of $g$.

Moreover

$$
\Omega(X, Y, Z)=g\left(\nabla_{X} Y-\bar{\nabla}_{X} Y, Z\right) .
$$

is the real part of a cubic holomorphic differential, where $\bar{\nabla}$ is the Levi-Civita connexion of $g$. .

We can translate the theorem in the language of splittings using the notations of Paragraph 2.2.2.

Theorem 4.2.2. [SPLITTING] Let $\Sigma$ be equipped with a convex projective structure. Let $E$ be the associated flat vector bundle equipped with a parallel volume form $\Omega$, then there exist a unique splitting $E=P \oplus \mathbb{R} u$ such that

- $\nabla^{u}$ defines the convex structure

- $\forall X, \nabla_{X} u \in P$.

- the volume form of the quadratic form $S_{u}$ is $\Omega\left(\nabla_{X} u, \nabla_{Y} u, u\right)$

4.2.1. Back to cubic holomorphic differentials. We first prove the second part of the Theorem.

Proposition 4.2.3. Let $(\nabla, g)$ be such that

- $\nabla^{g}$ is flat

- $\nabla$ preserves the area form of $g$.

Let $\nabla_{g}$ is the Levi-Civita connexion of $g$. Then

$$
\Omega(X, Y, Z)=g\left(\nabla_{X} Y-(\bar{\nabla})_{X} Y, Z\right)
$$

is the real part of a cubic holomorphic differential.

Proof : Let $A(X) Y=\nabla_{X} Y-\bar{\nabla}_{X} Y$. We leave to the reader to check that $\Omega$ is the real part of a cubic holomorphic differential if and only if

(1) $d^{\bar{\nabla}} A=0$

(2) $A(X) Y=A(Y) X$

(3) $A(X)$ is symmetric.

(4) $A(X)$ is trace free. 
Since $\bar{\nabla}$ preserves the area form of $g, A(X)$ is trace free . Thus Condition (4) is satisfied. Recall that by definition $\nabla^{g}$ is given by

$$
\nabla_{X}^{g}\left(\begin{array}{c}
Z \\
\lambda
\end{array}\right)=\left(\begin{array}{c}
\nabla_{X} Z+\lambda X \\
L_{X} \lambda+g(Z, X)
\end{array}\right)
$$

We compute various parts of the curvature tensor $R$ of $\nabla^{g}$. First,

$$
0=R(X, Y)\left(\begin{array}{l}
0 \\
1
\end{array}\right)
$$

yields

$$
\nabla_{X} Y-\nabla_{Y} X-[X, Y]=0 .
$$

Thus $\nabla$ is torsion free. Hence, Condition (2) is satisfied. Let us compute the other part of the curvature tensor

$$
0=R(X, Y)\left(\begin{array}{l}
Z \\
0
\end{array}\right)
$$

yields

$$
\left\{\begin{array}{r}
R^{\nabla}(X, Y) Z+g(Y, Z) X-g(X, Z) Y=0 \\
g(A(Y) Z, X)-g(A(X) Z, Y)=0
\end{array}\right.
$$

Using Condition (2), the second line of this equation reads

$$
g(A(Z) Y, X)=g(A(Z) X, Y) .
$$

Thus, Condition (3) is satisfied. The first line yields

$$
R^{g}(X, Y) Z+d^{\nabla^{g}} A(X, Y) Z+[A(X), A(Y)] Z+g(Y, Z) X-g(Z, X) Y=0 .
$$

However, the linear operator $Z \mapsto g(Y, Z) X-g(X, Z) Y$ is antisymmetric. The same holds for $R^{g}(X, Y)$ and $[A(X), A(Y)]$, since $A(X)$ is symmetric. Thus $Z \mapsto d^{\nabla} A(X, Y)(Z)$ is antisymmetric. However, $A(X)$ is symmetric, thus $Z \mapsto$ $d^{\nabla} A(X, Y)(Z)$ is symmetric. Therefore $d^{\nabla} A=0$ and Condition (1) is satisfied.Q.E.D.

4.2.2. Proof of Theorem 4.2.1. Let $\nabla^{0}$ be a flat connection on $E_{M}=M \otimes \mathbb{R}^{n+1}$ preserving a volume form $\Omega^{0}$. Let $u$ be a section of $E_{M}$ such that $\left(\nabla^{0}, u\right)$ give rise to the projective structure. It follows from Paragraph 2.2.2 that for every pair $(\nabla, g)$ where

- $g$ is positive,

- $\nabla$ equivalent to the convex structure,

- $\nabla$ preserves a volume form,

- $\nabla^{g}$ is flat,

there exists a function $f$ such that

$$
S_{\frac{u}{f}}=g
$$


Let now

For every $f \in \mathcal{U}$,

$$
\mathcal{U}=\left\{f \in C^{\infty}(\Sigma), \text { such that } S_{\frac{u}{f}} \text { is positive definite }\right\} .
$$

- Let $\omega^{f}$ be the area form of $S_{\frac{u}{f}}$.

- Let $\nu^{f}(X, Y)=\Omega^{0}\left(\nabla_{X} \frac{u}{f}, \nabla_{Y} \frac{u}{f}, \frac{u}{f}\right)$

The theorem will follow from the following proposition.

Proposition 4.2.4. The operator

is a diffeomorphism.

$$
D:\left\{\begin{array}{l}
\mathcal{U} \rightarrow C^{\infty}(\Sigma,] 0, \infty[) \\
f \mapsto \frac{\nu^{f}}{\omega^{f}}
\end{array}\right.
$$

4.2.3. Proof of Theorem 4.2.1 from Proposition 4.2.4. Let $\nabla=\nabla^{u}$ be the connection on $\Sigma$ given by

$$
\nabla_{X}^{0} \nabla_{Y}^{0} u=S_{u}(X, Y) u+\nabla_{\nabla_{X} Y}^{0} u .
$$

We first recall from Equation (3) of Proposition 2.2.3, that

$$
S_{\frac{u}{f}}=S_{u}-\frac{\nabla^{2} f}{f} .
$$

In particular, $S_{\frac{u}{k f}}=S_{\frac{u}{f}}$. Hence $D(k f)=k^{3} D(f)$. Now observe that $\omega_{f}$ is parallel for $\nabla^{\frac{u}{f}}$. It follows that the pairs $(\nabla, g)$ for which $\nabla \omega_{g}=0$, corresponds to the functions $f$, up to a multiplicative constant, such that $D(f)$ is constant. Thus, the first part of Theorem 4.2.1 follows from Proposition 4.2.4.

4.2.4. Proof of Proposition 4.2.4. Let $A(f)$ be the symmetric endomorphism defined by

$$
\nabla^{2} f(X, Y)=S_{u}(A(f) X, Y)
$$

Observe that $\nabla=\nabla_{u}$ is torsion free. Thus $A(f)$ is symmetric. Then

$$
\begin{aligned}
\nu^{f} & =\operatorname{det}\left(1-\frac{A(f)}{f}\right) \nu^{1} \\
& =\operatorname{det}\left(1-\frac{A(f)}{f}\right) f^{3} D(1) \omega^{f}
\end{aligned}
$$

Therefore

$$
D(f)=\operatorname{det}\left(f^{3 / 2}-f^{1 / 2} A(f)\right) D(1) .
$$

The proof of the Proposition will follows from the following three steps

(1) $D$ is a local diffeomorphism from $\mathcal{U}$ in $C^{\infty}(\Sigma] 0,,+\infty[)$,

(2) $D$ is proper,

(3) $\mathcal{U}$ is connected. 
This will require three separate propositions.

Proposition 4.2.5. $D$ is a local diffeomorphism from $\mathcal{U}$ in $C^{\infty}(\Sigma] 0,,+\infty[)$,

Proof : We first compute the linearised operator $L_{f}^{D}$ at $f$. Let

$$
G(f)=f^{3 / 2}-f^{1 / 2} A(f) .
$$

Then a straightforward computation give

$$
\begin{aligned}
L_{f}^{D}(\mu) & =\frac{D(1)}{D(f)} \operatorname{trace}\left(\left(\frac{3}{2} f^{\frac{1}{2}} \mu-\frac{1}{2} f^{-\frac{1}{2}} A(f) \mu-f^{\frac{1}{2}} A(\mu)\right) \circ G(f)^{-1}\right) \\
& \left.=\frac{D(1)}{D(f)} \operatorname{trace}\left(\mu\left(f^{\frac{1}{2}}+\frac{G(f)}{f}\right)-f^{\frac{1}{2}} A(\mu)\right) \circ G(f)^{-1}\right)
\end{aligned}
$$

Since $f \in \mathcal{U}, G(f)$ is a positive symmetric operator, it follows that $L_{f}^{D}$ is an elliptic operator. By the implicit function theorem, to prove the Proposition it suffices to show that $L_{f}^{D}$ is invertible. Since $L_{f}^{D}$ is homotopic to a Laplacian, its index is zero. It thus suffices to show that $L_{f}^{D}$ is injective. Let $\mu$ so that $L_{f}^{D}(\mu)=0$. Hence

$$
\operatorname{trace}\left(A(\mu) \circ G(f)^{-1}\right)=\mu \operatorname{trace}\left(\left(f^{1 / 2}+f^{-1} G(f)\right) \circ G\left(f^{-1}\right)\right)
$$

We apply the maximum principle: at a point where $\mu$ is maximum, $A(\mu)$ is nonpositive, hence trace $\left(A(\mu) \circ G(f)^{-1}\right)$ is nonpositive. We also know that $f^{1 / 2}+$ $f^{-1} G(f)$ is positive. It follows that trace $\left(\left(f^{1 / 2}+f^{-1} G(f)\right) \circ G\left(f^{-1}\right)\right)$ is positive. Hence Equation (11) implies that $\mu$ is nonpositive at its maximum. Symmetrically we prove that the minimum of $\mu$ is nonnegative. Hence $\mu=0$.

Q.E.D.

Proposition 4.2.6. The operator $D$ is proper.

Proof : According to the terminology used in the Appendix 12, $D$ is a Monge Ampère operator. To prove that $D$ is proper, by Proposition 12.0.6, it suffices to find a-priori bounds - depending on $g$ - for $f$ and its first derivatives whenever $D(f)=g$.

We first obtain $C^{0}$ bounds on $f$. Let $k_{1}=\inf (f)$. At a point where $f$ reaches its minimum, $A(f)$ is a positive operator. It follows that at this point we have

$$
D(f) \leq D\left(k_{1}\right)=k_{1}^{3} D(1) .
$$

Therefore

$$
\inf (f) \geq\left(\frac{\inf (g)}{\sup (D(1))}\right)^{\frac{1}{3}}
$$


A symmetric argument yields

$$
\sup (f) \leq\left(\frac{\sup (g)}{\inf (D(1))}\right)^{\frac{1}{3}} .
$$

We now obtain $C^{1}$ bounds on $f$ : We restrict a function $f$ of $\mathcal{U}$ on any geodesic for $\nabla$. Hence we obtain a function depending on one variable so that

$$
\ddot{f} \leq f .
$$

Since $f$ is bounded, this implies that $\dot{f}$ is also bounded. Therefore $f$ has $C^{1}$ bounds. Q.E.D.

Proposition 4.2.7. The set $\mathcal{U}$ is connected.

Proof : Indeed $\mathcal{U}$ is the set of functions $f$ such that

$$
f S_{u}-\nabla^{2} f
$$

is a positive symmetric tensor. It follows that $\mathcal{U}$ is convex, hence connected. Q.E.D.

\section{Projective structures and Cohomology Classes}

Let $\nabla$ be a flat connection on a rank 3 vector bundle $E$ over a surface $\Sigma$. We say a 1 -form $\alpha$ with values in $E$ is injective if

$$
\forall X \in T \Sigma \backslash\{0\}, \quad \alpha(X) \neq 0 .
$$

Every injective closed 1-form $\alpha$ with values in $E$ defines a section $v=\alpha(T \sigma)$ of $\mathbb{P}\left(E^{*}\right)$. Moreover, an injective closed form $\alpha$ defines a symmetric bilinear tensor up to a multiple on $T \Sigma$ by

$$
h_{\alpha}^{\nabla}(X, Y)=\pi\left(\nabla_{X}(\alpha(Y))\right) .
$$

where $\pi$ is the projection of $E$ on the rank-1 bundle $E / \alpha(T \Sigma)$. If $h_{\alpha}^{\nabla}$ is nondegenerate, then $v$ defines an equivariant immersion, hence a projective structure $\mathfrak{p}$ on $\Sigma$.

We say that $\omega$ is convex if for every nonzero vector $h_{\omega}^{\nabla}(X, X) \neq 0$. We say the complex structure $J$ is compatible with the convex form $\omega$ if it defines the conformal class of $\omega$ that is

$$
h_{\omega}^{\nabla}(X, X)=h_{\omega}^{\nabla}(J X, J X) .
$$

From the previous observation a convex 1-form also defines a projective structure.

Let $\mathcal{O}_{\mathfrak{p}}$ be the open cone of cohomology classes of convex 1-forms in $H^{1}(E)$ defining $\mathfrak{p}$. We shall prove the following theorem 
Theorem 5.0.8. Let $\mathfrak{p}$ be a projective structure. Let $J$ be a complex structure on $\Sigma$. Then, there exists a convex 1-form defining $\mathfrak{p}$ and compatible with $J$. As a consequence $\mathcal{O}_{\mathfrak{p}}$ is nonempty.

Finally the projective structure $\mathfrak{p}$ is convex if and only if $\mathcal{O}_{\mathfrak{p}}$ contains 0 .

\section{REMARKS}

- It follows that if $\mathfrak{p}$ is convex, then $\mathcal{O}_{\mathfrak{p}}=H^{1}(E)$.

- This result leads to the following natural set of questions:

(1) given $\mathfrak{p}$ and $J$ does there exist a better convex form defining $\mathfrak{p}$ and compatible with $J$ ? A positive answer to this question would lead to a parametrisation of $\mathcal{O}_{\mathfrak{p}}$ by Teichmüller space.

(2) given $\mathfrak{p}$ and $\omega$ in $\mathcal{O}_{\mathfrak{p}}$ does there exist a better complex structure $J$ such that $\omega$ can be represented by a convex form defining $\mathfrak{p}$ and compatible with $J$ ?

A positive answer to these questions would lead to a map parametrising $\mathcal{O}_{\mathfrak{p}}$ by Teichmüller space. In Theorem 6.0 .11 , when $\mathfrak{p}$ is convex, we actually produce a map from $H^{1}(E)$ to Teichmüller space.

5.0.5. A preliminary proposition. We begin with a proposition

Proposition 5.0.9. Let $\alpha$ be an injective convex form on $T \Sigma$ with values in $E$ equipped with a flat connection $\nabla$. Then, the geodesics for the associated projective structure are the curves $c$ so that there exist a vector field $Y$ along $c$ so that

$$
\nabla_{\dot{c}} \alpha(Y)=0 \text {. }
$$

Proof : This is a local statement. Therefore, we can assume that the connection $\nabla$ is the trivial one and we identify sections of the vector bundle as maps with values in a vector space. Then, a curve $c$ is a geodesic if and only if, its image under $v=\alpha(T \Sigma)$ is a dual projective line. This means that all the planes $\alpha(T \Sigma)$ contain a common nonzero vector $Z$. Therefore, if $Y$ is the vector field along $c$ such that $\alpha(Y)=Z$, it follows that $\alpha(Y)$ is a parallel section of $E$ along c. Q.E.D.

5.0.6. Proof of Theorem 5.0.8. Proof : Let $J$ be a complex structure on $\Sigma$. Let $\mathfrak{p}$ be a projective structure on $\Sigma$. Let $(\nabla, h)$ be a pair consisting of a torsion free connection $\nabla$ preserving a volume form $\omega$ and representing $\mathfrak{p}$, and $h$ a symmetric tensor so that $\nabla^{h}$ is flat. Recall that $\nabla^{h}$ is a connection on $E=T \Sigma \oplus \mathbb{R}$. Let $g$ be the metric on $T \Sigma$ given by $g(X, X)=\omega(X, J X)$. We equip $E$ with the metric given by

$$
G((X, \lambda),(X, \lambda))=g(X, X)+\lambda^{2} .
$$


Let $\nabla^{h, *}$ (respectively $\nabla^{*}$ ) be the dual connection to $\nabla^{h}$ with respect to $G$ (respectively $g)$. Let $H$ be the symmetric tensor so that $g(H X, Y)=h(X, Y)$. Observe that

$$
\nabla_{Z}^{h, *}(X, \lambda)=\left(\nabla_{Z}^{*} X+\lambda H(Z), g(X, Z)+d \lambda(Z)\right) .
$$

Let $\alpha \in \Omega^{1}(T \Sigma) \otimes E$ be given by $\alpha(X)=(X, 0)$. Observe that $d^{\nabla^{h, *}} \alpha=0$. Moreover,

$$
h_{\alpha}^{\nabla^{*}}=\pi\left(\nabla^{h, *} X Y\right)=g(X, Z) .
$$

Hence $\alpha$ is convex (with respect to the connection $\nabla^{h, *}$ ). Let $\mathfrak{q}$ be the associated projective structure. By Proposition 5.0.9, the geodesics for $\mathfrak{q}$ are those curves $c$ along which there exist a vector field $Y$ such that $\alpha(Y)$ is parallel. Therefore we have

$$
0=\nabla_{\dot{c}}^{h, *}(Y, 0)=\left(\nabla_{\dot{c}}^{*} Y, g(\dot{c}, Y)\right)
$$

This is equivalent to the collinearity of $\nabla_{\dot{c}}^{*} J \dot{c}$ with $J \dot{c}$. Since, $\nabla^{*}=-J \nabla J$, this is equivalent to $c$ being a geodesic for $\nabla$. Hence, $\mathfrak{q}=\mathfrak{p}$. This finishes the proof of the first part of the Theorem.

Finally, if $0 \in \mathcal{O}_{\mathfrak{p}}$, this means that we can find a section $u$ so that $\nabla u$ is an injective form defining $\mathfrak{p}$, and $S_{u}$ is positive definite. Hence $\nabla^{u}$ defines a convex structure (see the definitions and notations in Paragraph 2.2.2)

By Proposition 5.0.9, a curve $c$ is a geodesic for $\mathfrak{p}$ if there exists a nonzero vector field $Y$ along $c$ so that

$$
\nabla_{\dot{c}} \nabla_{Y} u=0 .
$$

This means that $\nabla_{\dot{c}}^{u} Y=0$ and $S_{u}(\dot{c}, Y)=0$. This means that $c$ is a geodesic for the connection $\nabla^{u, *}$ dual to $\nabla^{u}$ with respect to $S_{u}$.

Finally, we notice that $\nabla^{u, *}$ also defines a convex structure. Indeed, by the previous discussion, the connection $\nabla^{*}=\left(\nabla^{u, *}\right)^{S_{u}}$ is dual to the connection $\nabla=\left(\nabla^{u}\right)^{S_{u}}$ with respect to the metric

$$
G((X, \lambda),(X, \lambda))=S_{u}(X, X)+\lambda^{2} .
$$

Thus $\nabla^{*}$ is flat and $\nabla^{u, *}$ is convex. We have finished proving that $\mathfrak{p}$ is convex. Q.E.D.

\section{Cohomology Classes and COMplex structures}

Our aim is to give a description of $H_{\rho}^{1}(E)$ in terms of complex structures on the surface when $\rho$ is the holonomy of a convex projective surface.

Theorem 6.0.10. [COMPLEX STRUCTURES] Let $\mathfrak{p}$ be a convex projective structure of holonomy $\rho$ on a closed surface $\Sigma$. Let $\nabla$ be the volume preserving 
connection and $J_{0}$ be the complex structure described by Theorem 4.2.1. Let $\nabla^{*}=-J_{0} \nabla J_{0}$ be the dual connection. Let

$$
\mathcal{J}=\left\{J \in \Gamma(\operatorname{End}(T \Sigma)) / J^{2}=-1 \text { and } d^{\nabla^{*}} J=0\right\} .
$$

Then the map from $\mathcal{J}$ to $H_{\rho}^{1}(E)$ given by

$$
J \rightarrow J_{0} J \in \Gamma(\operatorname{End}(\mathrm{T} \Sigma))=\Omega^{1}(\Sigma) \otimes T \Sigma \subset \Omega^{1}(\Sigma) \times E,
$$

is a bijection.

Notice that when $\rho$ takes values in $S O(2,1)$, the canonical map from $\mathcal{J}$ to Teichmüller space is an isomorphism. This follows from the fact that $d^{\nabla} J=0$ if and only if the identity map is an harmonic mapping from $\Sigma$ equipped with $J$ to $\Sigma$ equipped with $\nabla$.

The Theorem is given by Proposition 6.1.

We also prove a linear version of this Theorem which gives another interpretation of $H_{\rho}^{1}(E)$

Theorem 6.0.11. [Hodge REPRESENTATIVEs] Let $\mathfrak{p}$ be a convex projective structure of holonomy $\rho$ on a closed surface $\Sigma$. Let $\nabla$ be the volume preserving connection and $J_{0}$ be the complex structure described by Theorem 4.2.1. Let

$$
\mathcal{H}_{J_{0}}=\left\{A \in \Gamma(\operatorname{End}(T \Sigma)) / A J_{0}=-J_{0} A \text { and } d^{\nabla} A=0\right\} .
$$

Then the map from $\mathcal{H}_{J_{0}}$ to $H_{\rho}^{1}(E)$ given by the inclusion

$$
\mathcal{H}_{J_{0}} \subset \Gamma(\operatorname{End}(\mathrm{T} \Sigma))=\Omega^{1}(\Sigma) \otimes T \Sigma \subset \Omega^{1}(\Sigma) \otimes E,
$$

is a bijection.

The theorem is given by Proposition 6.2.1.

We observe that when $\rho$ takes values in $S O(2,1)$, there is a bijection between $\mathcal{H}_{J_{0}}$ and the space of quadratic holomorphic differentials and we recover here a case of the Eichler-Shimura isomorphism.

In the next section, we explain how these results yield interesting symmetries between representations in the affine group.

6.1. Cohomology classes and complex structures. Our aim is to prove the following Proposition which generalises Theorem 6.0.10.

Proposition 6.1.1. Let $\mathfrak{p}$ be a convex projective structure on $\Sigma$ of holonomy $\rho$. Let $\nabla$ be a connection representing $\mathfrak{p}$. Assume there exist a metric $g$ such that $\nabla^{g}$ on $E=T \Sigma \oplus \mathbb{R}$ is flat. Let $J_{0}$ be the complex structure of $g$. Let $\tilde{\nabla}=-J_{0} \nabla J_{0}$. Let $\mu$ be a an element of $H_{\rho}^{1}(E)$. Then there exists a unique complex structure $J$ on $\Sigma$ such that

$$
d^{\tilde{\nabla}} J=0,
$$


and

$$
J_{0} J \in \Omega^{1}(\Sigma) \otimes T \Sigma \subset \Omega^{1}(\Sigma) \otimes E,
$$

has cohomology class $\mu$.

We observe that in the case $\nabla$ preserves the volume form of $g$ (i.e. we are in the case described by Theorem 4.2.1), then $\tilde{\nabla}$ is the dual connection of $\nabla$ with respect to $g$.

6.1.1. A Monge-Ampère equation. Let $g$ be a metric on $\Sigma$. Let $B_{\mu}$ be any symmetric operator on $T \Sigma$. Let $\nabla$ be any connection on $\Sigma$. Let

$$
H_{\mu}:\left\{\begin{aligned}
C^{\infty}(\Sigma) & \rightarrow C^{\infty}(\Sigma) \\
f & \left.\mapsto \operatorname{det}\left(B_{\mu}+f-\nabla^{2} f\right)\right\}
\end{aligned}\right.
$$

We now prove

Proposition 6.1.2. Let $\mathcal{U}_{\mu}=\left\{f \in C^{\infty}(\Sigma) / H_{\mu}(f)>0\right\}$. Then $H_{\mu}$ is a diffeomorphism from $\mathcal{U}_{\mu}$ to $C^{\infty}(\Sigma] 0,, \infty[)$.

6.1.2. Proof of Proposition 6.1.2. This proof will follow closely the strategy of the proof of Proposition 4.2.4. We use the following three steps

(1) $H_{\mu}$ is a local diffeomorphism from $\mathcal{U}_{\mu}$ in $C^{\infty}(\Sigma] 0,,+\infty[)$,

(2) $H_{\mu}$ is proper,

(3) $\mathcal{U}_{\mu}$ is connected.

This will require three separate propositions.

Proposition 6.1.3. $H_{\mu}$ is a local diffeomorphism from $\mathcal{U}_{\mu}$ in $C^{\infty}(\Sigma] 0,,+\infty[)$,

Proof : We first compute the linearised operator $L_{f}^{H_{\mu}}$ at $f$ of $H_{\mu}$. Let

$$
G(f)=B_{\mu}+f-A(f) .
$$

Then, we get

$$
L_{f}^{H_{\mu}}(g)=\frac{1}{H_{\mu}(f)} \operatorname{trace}\left((g-A(g)) \circ G(f)^{-1}\right)
$$

Since $f \in \mathcal{U}_{\mu}, G(f)$ is a positive symmetric operator, hence $L_{f}^{H_{\mu}}$ is an elliptic operator. By the implicit function theorem, to prove Proposition 6.1.3 it suffices to show that $L_{f}^{H_{\mu}}$ is invertible. Since $L_{f}^{H_{\mu}}$ is homotopic to a Laplacian, its index is zero. It thus suffices to show that $L_{f}^{H_{\mu}}$ is injective. Let $g$ so that $L_{f}^{H_{\mu}}(g)=0$. Hence

$$
\operatorname{trace}\left(A(g) \circ G(f)^{-1}\right)=g \operatorname{trace}\left(G\left(f^{-1}\right)\right)
$$


We apply the maximum principle: at a point where $g$ is maximum, $A(g)$ is nonpositive, hence trace $\left(A(\mu) \circ G(f)^{-1}\right)$ is nonpositive. We also know that $G(f)$ is positive. It follows that trace $\left(G\left(f^{-1}\right)\right)$ is positive. Hence Equation (12) implies that $g$ is nonpositive at its maximum. Symmetrically we prove that the minimum of $g$ is nonnegative. Hence $g=0$.

Q.E.D.

Proposition 6.1.4. The operator $H_{\mu}$ is proper.

Proof : According to the terminology used in the Appendix $12, H_{\mu}$ is a Monge-Ampère operator. To prove that $H_{\mu}$ is proper, by Proposition 12.0.6, it suffices to find a-priori bounds - depending on $g$ - for $f$ and its first derivatives whenever $H_{\mu}(f)=g$.

We first obtain $C^{0}$ bounds on $f$. Let $k_{1}=\inf (f)$. At a point where $f$ reaches its minimum, $A(f)$ is a positive operator. Let in general $\Lambda_{\mu}$ (respectively $\lambda_{\mu}$ ) be the greatest (respectively smallest) eigenvalue of $B_{\mu}$. Hence, at a minimum of $f$, we have

$$
H_{\mu}(f) \leq H_{\mu}\left(k_{1}\right) \leq\left(k_{1}+\Lambda_{\mu}\right)^{2} .
$$

Therefore

$$
\inf (f) \geq \inf (g)^{\frac{1}{2}}-\Lambda_{\mu}
$$

A symmetric argument yields

$$
\sup (f) \leq \sup (g)^{\frac{1}{2}}-\lambda_{\mu} .
$$

We now obtain $C^{1}$ bounds on $f$. Let $f \in \mathcal{U}_{\mu}$. Then the function $f$ restricted to any geodesic satisfies

$$
\ddot{f} \leq f+h
$$

for some function $f$. Now, $C^{0}$-bounds on $f$ implies bounds on $\dot{f}$. It follows that any $f$ such that $H_{\mu}(f)=g$ admits $C^{1}$-bounds. Q.E.D.

Proposition 6.1.5. The set $\mathcal{U}_{\mu}$ is connected.

Proof : Indeed $\mathcal{U}_{\mu}$ is the set of functions $f$ such that

$$
B_{\mu}+f-\nabla^{2} f,
$$

is a a positive symmetric tensor. It follows that $\mathcal{U}_{\mu}$ is convex, hence connected. Q.E.D.

6.1.3. Proof of Proposition 6.1. Let $\mu$ be a a closed 1-form in $\Omega^{1}(\Sigma) \otimes E$. We write

$$
\mu(X)=\left(B_{\mu}(X), \alpha_{\mu}(X)\right) \in T \Sigma \oplus \mathbb{R}=E,
$$


where $B_{\mu} \in \operatorname{End}(T \Sigma)$ and $\alpha \in T^{*}(\Sigma)$. Let $\xi_{\mu}$ be the vector field such that $g\left(\xi_{\mu}, X\right)=\alpha_{\mu}(X)$. Then

$$
\alpha_{\mu-\nabla \xi_{\mu}}=0
$$

It follows that every cohomology class in $E$ has a representative $\omega$ such that $\alpha_{\omega}=0$.

Let $\omega$ be such a representative. Let $v=(\xi, f)$ be a section of $E$. We observe also that

$$
\alpha_{\omega+\nabla v}=0
$$

if and only if $\xi=-\operatorname{grad} f$. Let $\nabla_{*}$ be the dual connection to $\nabla$ with respect to $g$, observe that

$$
\nabla \operatorname{grad} f=\nabla_{*}^{2} f
$$

Let $v_{f}=(-\operatorname{grad} f, f)$. We have

$$
B_{\omega+\nabla v_{f}}=B_{\omega}+f-\nabla_{*}^{2} f .
$$

By Proposition 6.1.2, we conclude there exists a unique representative $\omega$ of the cohomology class $\mu$ such that $\alpha_{\omega}=0$ and $\operatorname{det}\left(B_{\omega}\right)=1$.

Proposition 6.1 follows from Proposition 6.1.3 and the following two observations

- if $B \in \Omega^{1}(\Sigma) \otimes T \Sigma \subset \Omega^{1}(\Sigma) \otimes E$ is closed, then $d^{\nabla} B=0$ and $B$ is symmetric.

- $B$ is symmetric with determinant equal to 1 , if and only if $J_{0} B$ is a complex structure.

6.2. Hodge representatives. We now prove the first part Theorem 6.0.11

Proposition 6.2.1. Let $\nabla$ be the torsion free connection and $g$ a metric such that $\nabla^{g}$ is flat of holonomy $\rho$. Let $J_{0}$ be the complex structure of $g$. Let

$$
\mathcal{H}_{J_{0}}=\left\{A \in \Gamma(\operatorname{End}(T \Sigma)) / A J_{0}=-J_{0} A \text { and } d^{\nabla} A=0\right\} .
$$

Then the map from $\mathcal{H}_{J_{0}}$ to $H_{\rho}^{1}(E)$ which factors through the inclusion

$$
\mathcal{H}_{J_{0}} \subset \Gamma(\operatorname{End}(\mathrm{T} \Sigma))=\Omega^{1}(\Sigma) \otimes T \Sigma \subset \Omega^{1}(\Sigma) \otimes E,
$$

is a bijection.

Proof : We shall use the notations of the proof of Proposition 6.1. Let $\mu$ be a cohomology class in $H_{\rho}^{1}(E)$. Using the same approach, we can represent $\mu$ by a 1 -form $\omega$ such that $\alpha_{\omega}=0$. We now remark there exists a unique function $f$ such that

$$
D(f)=\operatorname{trace}\left(f-\nabla_{*}^{2} f\right)=-\operatorname{trace}\left(B_{\omega}\right) .
$$

Indeed, $D$ is a linear elliptic operator of index 0 whose kernel is trivial as it is shown by an easy application of the maximum principle. 
We also remark that $A$ is symmetric of trace zero if and only if $A J_{0}=-J_{0} A$. Combining these two remarks, we obtain that there exists a unique section $v$ of $E$ such that $\beta=\omega+\nabla v$ satisfies

$$
\begin{aligned}
\alpha_{\beta} & =0 \\
B_{\beta} J_{0} & =-J_{0} B_{\beta} .
\end{aligned}
$$

The statement follows Q.E.D.

\section{Dualities AND Symmetries of MOduli sPaCeS}

We now explain that Theorems 4.2.1, 6.0.10 and 6.0.11, give rise to interesting symmetries of moduli spaces of representations, well known in the first case, but more mysterious in the other cases.

7.1. Contragredient representation and Theorem 4.2.1. We define $\operatorname{Rep}_{\mathrm{H}}\left(\pi_{1}(\Sigma), P S L(3, \mathbb{R})\right)$ to be the component of the space of representations which contains the fuchsian faithful and discrete - representations in $S O(2,1)$. Let $S A f f(3, \mathbb{R})$ be the special affine group in dimension 3

$$
\operatorname{SAff}(3, \mathbb{R})=\mathbb{R}^{3} \rtimes \operatorname{PSL}(3, \mathbb{R}) .
$$

We define similarly $\operatorname{Rep}_{H}\left(\pi_{1}(\Sigma), S A f f(3, \mathbb{R})\right)$ to be the set of those representations whose linear part is in $\operatorname{Rep}_{\mathrm{H}}\left(\pi_{1}(\Sigma), P S L(3, \mathbb{R})\right)$. We observe that by results of Choi and Goldman [4], $\operatorname{Rep}_{\mathrm{H}}\left(\pi_{1}(\Sigma), P S L(3, \mathbb{R})\right)$ is precisely the set of monodromies of convex projective structures.

Let $\omega$ be a volume form on $\Sigma$. By our Theorem 4.2.1, it follows that

$$
\operatorname{Rep}_{\mathrm{H}}\left(\pi_{1}(\Sigma), P S L(3, \mathbb{R})\right.
$$

is in one-to-one correspondence with the space of triples $(\nabla, \omega, J)$ where $\nabla$ is connection, $\omega$ is a volume form, and $J$ is a complex structure, which satisfy condition $(\mathrm{H})$ :

$$
\left\{\begin{aligned}
\nabla_{X} Y-\nabla_{Y} X & =[X, Y] \\
\nabla \omega & =0 \\
d \nabla J & =0 \\
R^{\nabla}(X, Y) J Z & =\omega(X, Y) Z .
\end{aligned}\right.
$$

It is a trivial observation that $(\nabla, \omega, J)$ satisfies condition $(\mathrm{H})$ if and only if $(-J \nabla J, \omega, J)$ does. The corresponding duality in the space of representations is the duality which associates to a representation its contragredient representation as it is shown by an easy exercise left to the reader. Its fixed-point set is the space of representations with monodromy in $S O(2,1)$. 
7.2. Theorem 6.0.10 and an involution on the moduli space of representations in the affine group. Theorem 6.0.10 provides a more mysterious duality. By this result,

$$
\operatorname{Rep}_{H}\left(\pi_{1}(\Sigma), S A f f(3, \mathbb{R})\right)
$$

is in bijection with space of quadruples $\left(\nabla, \omega, J, J_{1}\right)$ where $\nabla$ is a connection $\omega$ a volume form, and $J$ as well as $J_{1}$ are complex structures, which satisfy condition (I):

$$
\left\{\begin{aligned}
\nabla_{X} Y-\nabla_{Y} X & =[X, Y] \\
\nabla \omega & =0 \\
d^{\nabla} J & =0 \\
R^{\nabla}(X, Y) J Z & =\omega(X, Y) Z \\
d^{\nabla} J J_{1} & =0
\end{aligned}\right.
$$

An exercise shows that $\left(\nabla, \omega, J, J_{1}\right)$ satisfies condition (I) if and only if

$$
\left(-J_{1} \nabla J_{1}, \omega,-J_{1} J J_{1}, J_{1}\right)
$$

does. We obtain therefore a duality on $\operatorname{Rep}_{\mathrm{H}}\left(\pi_{1}(\Sigma), S A f f(3, \mathbb{R})\right)$ which extends the duality on $\operatorname{Rep}_{\mathrm{H}}\left(\pi_{1}(\Sigma), P S L(3, \mathbb{R})\right)$ considered as a subset. However this duality does not respect the projection from $\operatorname{Rep}_{\mathrm{H}}\left(\pi_{1}(\Sigma), S A f f(3, \mathbb{R})\right)$ to $\operatorname{Rep}_{\mathrm{H}}\left(\pi_{1}(\Sigma), P S L(3, \mathbb{R})\right)$ and does not seem to have an algebraic description. Again, its fixed-point set is the space of representations with monodromy in $S O(2,1)$.

\subsection{Theorem 6.0.11 and a fourth order symmetry on the moduli space} of representations in the affine group. Finally, Theorem 6.0.11 also provides a symmetry, of order 4 , on

$$
\operatorname{Rep}_{H}\left(\pi_{1}(\Sigma), S A f f(3, \mathbb{R})\right) .
$$

By this result, this moduli space bijects with space of quadruples $(\nabla, \omega, J, A)$ where $\nabla$ is a connection, $\omega$ a volume form, $J$ is complex structure and $A$ is an endomorphism of $T \Sigma$, which satisfy condition $(\mathrm{J})$ :

$$
\left\{\begin{aligned}
\nabla_{X} Y-\nabla_{Y} X & =[X, Y] \\
\nabla \omega & =0 \\
d^{\nabla} J & =0 \\
R^{\nabla}(X, Y) J Z & =\omega(X, Y) Z . \\
d^{\nabla} A & =0 \\
A J & =-J A
\end{aligned}\right.
$$

Again, it is an exercise that $(\nabla, J, A)$ satisfy condition $(\mathrm{J})$ if and only if $(-J \nabla J, J, J A)$ does. Observe that the map

$$
j:(\nabla, J, A) \rightarrow(-J \nabla J, J, J A),
$$


is actually of order $4: j^{2}$ sends $(\nabla, J, A)$ to $(\nabla, J,-A)$ and is the antipodal map on the vector bundle

$$
\operatorname{Rep}_{\mathrm{H}}\left(\pi_{1}(\Sigma), S A f f(3, \mathbb{R})\right) \rightarrow \operatorname{Rep}_{\mathrm{H}}\left(\pi_{1}(\Sigma), P S L(3, \mathbb{R})\right) .
$$

This mapping $j$ extends the duality of $\operatorname{Rep}_{H}\left(\pi_{1}(\Sigma), P S L(3, \mathbb{R})\right.$ as a subset and also factors over the projection to $\operatorname{Rep}_{H}\left(\pi_{1}(\Sigma), P S L(3, \mathbb{R})\right.$.

\section{AN AFFINE DIFFERENTIAL INTERPRETATION}

In this section we interpret Theorems 4.2.1, 6.0.10 in terms of affine differential geometry. We also give an interpretation of Theorem 5.0.8 in this language.

Recall first the description of convex hypersurfaces in affine differential geometry. Let $E$ be an affine space equipped with a constant volume form $\Omega$. We denote by $D$ its connection. Let $\Sigma$ be a locally convex hypersurface in $E$. Then there exists a unique pair $(g, \nu)$ such that

- $\nu$ is a vector field along $\Sigma$ transverse to $T \Sigma$,

- $g$ is a metric on $\Sigma$ whose volume form is $i_{\nu} \omega$,

- for all $X$ in $T \Sigma$, we have $\nabla_{X} \nu \in T \Sigma$,

- for all $X, Y$ in vector fields on $\Sigma$ we have $D_{X} Y-g(X, Y) \nu \in T \Sigma$.

The vector field is the affine normal vector field and $g$ is the Blaschke metric. We call $B=\nabla \nu$ the affine shape operator and $\nabla$ is the Blaschke connection

In other words, if we decompose

$$
\left.T E\right|_{\Sigma}=T \Sigma \oplus \mathbb{R} \nu
$$

Then the connection $D$ on $\Sigma$ decomposes as

$$
D_{X}(Y, \lambda)=\left(\nabla_{X} Y+\lambda B(X), g(X, Y)+d \lambda(X)\right) .
$$

If $\bar{\nabla}$ is the Levi-Civita connection of the metric $g, \nabla-\nabla_{g}$ is the Pick invariant $P$. Conversely, if $\Sigma$ is simply connected, if $B, \nabla$ and $g$ satisfy the following conditions

- $\nabla$ is torsion free, preserves the volume form of $g$

- the connection $D$ defined by Equation (15) is flat, that is

$$
\left\{\begin{aligned}
g(B X, Y) & =g(X, B Y) \\
d^{\nabla} g & =0 \\
d^{\nabla} B & =0 \\
R^{\nabla}(X, Y) Z & =g(X, Z) B Y-g(Y, Z) B X
\end{aligned}\right.
$$

then there exists an immersion of $\Sigma$ whose Blaschke metric is $g$, shape operator is $B$ and Blaschke connection is $P$. If $\Sigma$ is not simply connected, then the universal cover of $\Sigma$ possesses an immersion equivariant under a representation 
in the special affine group. The linear part of this representation is given by the holonomy $\rho_{0}$ of $D$, and the affine extension as en element of $H_{\rho_{0}}^{1}\left(\pi_{1}(\Sigma), E\right)=$ $H_{D}^{1}(T \Sigma \oplus \mathbb{R})$ is represented by the element $\omega$ of $\Omega^{1}(\Sigma) \otimes(T \Sigma \oplus \mathbb{R})$ given by $\omega(X)=(X, 0)$.

We concentrate now on the case of surfaces and write every metric $g$ as $g=$ $\omega(., J)$, where $\omega$ is the volume form of $g$ and $J$ is the complex structure of $g$. We write now the equations on $\nabla, \omega, J$ and $B$ which translates the condition (16) above

$$
\left\{\begin{aligned}
\nabla_{X} Y-\nabla_{Y} X & =[X, Y] \\
\nabla \omega & =0 \\
\operatorname{trace}(B J) & =0 \\
d^{\nabla} J & =0 \\
d^{\nabla} B & =0 \\
R^{\nabla}(X, Y) Z & =-\omega(X, Y) B J Z
\end{aligned}\right.
$$

8.1. Hyperbolic affine spheres and Theorem 4.2.1. An affine sphere is such that $B=k \mathrm{Id}$. For $k=-1$, we say the affine sphere is elliptic, for $k=0$ we say it is parabolic, for $k=1$, we say it is hyperbolic. There are strong relations between strictly convex cones and hyperbolic affine spheres as explained by the following difficult theorem conjectured by Calabi [1]. This result is due to Cheng and Yau [2] and [3] later completed and clarified by the work of Gigena [6], Sasaki [20] and A.M. Li [17], [18].

Theorem 8.1.1. [CHENG-YAu] [HYPERBOLIC AFFInE SPHERES BOUND CONES] If $\Sigma$ is a hyperbolic affine sphere with a complete Blaschke metric, then $\Sigma$ is properly embedded and bounds a convex cone. Conversely any strictly convex cone is asymptotic to such a unique hyperbolic affine sphere.

In the case where $E$ is of dimension 3, it follows from this result that for every convex structure one can associate an hyperbolic affine sphere invariant under the monodromy of the convex structure: the affine sphere asymptotic to the convex set of $\mathbb{P}(E)$ on which the monodromy acts cocompactly. This is precisely the content of Theorem 4.2.1. However, our proof uses a simpler approach.

The relation of Theorem 4.1.1, between the cubic holomorphic form and the Pick invariant is a Theorem of C.-P. Wang [24] as we already said.

8.2. Constant Gaussian curvature surfaces and Theorem 6.0.10. We suppose again that $E$ has dimension 3. We consider now constant Gaussian curvature 1 affine hypersurfaces (or CCG hypersurfaces) namely those surfaces for which $\operatorname{det} B=1$ where $B$ is the affine shape operator. Therefore, we can write $B=J J_{1}$ where $J_{1}$ is a complex structure on $\Sigma$. 
It follows from an easy check that $\left(\nabla, \omega, J, B=J J_{1}\right)$ satisfies Condition 17 , if and only if $\left(-J \nabla J, J_{1}, J\right)$ satisfies Condition 14 . In other words, we can restate Theorem 6.0.10 using this observation in the following way.

Theorem 8.2.1. Let $\Sigma$ be a compact surface. Given any representation $\rho$ element of $\operatorname{Rep}_{\mathrm{H}}\left(\pi_{1}(\Sigma), S A f f(3, \mathbb{R})\right.$, there exists a unique $C C G$ invariant under this representation.

This result does not seem to be known in the affine differential world.

8.3. Interpretation of Theorem 5.0.8. Every locally convex surface $\Sigma$ in affine space admits a natural projective structure: the one given by the immersion $s \mapsto T_{s} \Sigma$. Whenever $\Sigma$ is equivariant under a representation $\rho$, the holonomy $\dot{\rho}$ of flat connection $D$ on $T \Sigma \oplus \mathbb{R}$ described by Formula 15 is the linear part of $\rho$. Moreover, the element $\omega$ of $\Omega^{1}(T \Sigma) \otimes(T \Sigma \oplus \mathbb{R})$ defined by $\omega(X)=(X, 0)$ is a representative in $H_{\rho_{0}}^{1}\left(\mathbb{R}^{3}\right)$ of the cohomology class describing the extension from $\rho_{0}$ to $\rho$. Moreover $\omega$ is convex in the sense of Section 5. Conversely, every convex closed 1 -form is obtained this way.

Therefore we can reinterpret Theorem 5.0.8 in the following way.

Theorem 8.3.1. Let $J$ be a complex structure on $\Sigma$. Let $\mathfrak{p}$ be a projective structure on $\Sigma$, with monodromy $\rho_{0}$.

Then there exists a locally convex surface $\Sigma$ in the affine three dimensional space whose Blaschke metric is conformal to $J$ and which defines $\mathfrak{p}$ equivariant under a representation $\rho$ whose linear part is $\rho_{0}$.

Moreover, we can choose $J$ so that $\rho$ is conjugate to $\rho_{0}$ if and only if $\mathfrak{p}$ is convex.

\section{A Higgs Bundle interpretation}

We now recall briefly the work of Hitchin on representations of surface groups in $P S L(n, \mathbb{R})$ and explain using [16] how it fits with the present work.

9.1. Representations and harmonic mappings. Following [15], we define a Fuchsian representation of $\pi_{1}(\Sigma)$ in $\operatorname{PSL}(n, \mathbb{R})$ to be a representation which factors through the irreducible representation of $\operatorname{PSL}(2, \mathbb{R})$ in $\operatorname{PS} L(n, \mathbb{R})$ and a cocompact representation of $\pi_{1}(\Sigma)$ in $\operatorname{PSL}(2, \mathbb{R})$. A Hitchin representation is a representation which may be deformed in a Fuchsian representation. The space of Hitchin representation is denoted by

$$
\operatorname{Rep}_{H}\left(\pi_{1}(\Sigma), P S L(n, \mathbb{R})\right)
$$

and is called a Hitchin component. 
In his article [8], N. Hitchin gives explicit parametrisations of Hitchin components. Namely, given a choice of a complex structure $J$ over a given compact surface $\Sigma$, he produces a homeomorphism

$$
H_{J}: \mathcal{Q}(2, J) \oplus \ldots \oplus \mathcal{Q}(n, J) \rightarrow \operatorname{Rep}_{H}\left(\pi_{1}(\Sigma), P S L(n, \mathbb{R})\right),
$$

where $\mathcal{Q}(p, J)$ denotes the space of holomorphic $p$-differentials on the Riemann surface $(\Sigma, J)$. The main idea in the proof is first to identify representations with harmonic mappings as in K. Corlette's seminal paper [9], (see also [11]), second to use the fact a harmonic mapping $f$ taking values in a symmetric space gives rise to holomorphic differentials in manner similar to that in which a connection gives rise to differential forms in Chern-Weil theory (cf. Paragraph 7.1.2 of [16]). We explain quickly the construction. Namely, we have a parallel symmetric $p$ form $q_{p}$ on $M=P S L(n, \mathbb{R}) / S O(n, \mathbb{R})$. Identifying (after a choice of a base point) $M$ with the space of metrics of volume 1 and $T_{g} M$ with the space of self-adjoint (with respect to $g$ ), endomorphism of $\mathbb{R}^{n}$ we set

$$
\left(q_{p}\right)_{g}(A, \ldots, A)=\operatorname{trace}(\underbrace{A \ldots A}_{p \text { times }}) .
$$

Then we can complexify $q_{p}$ as a parallel symmetric complex $p$ form on the complexified tangent bundle. Then, the $p$-ic holomorphic form $Q_{p}(f)$ associated to a harmonic mapping $f$ with values in $M=P S L(n, \mathbb{R}) / S O(n, \mathbb{R})$ is

$$
Q_{p}(f)=q_{p}(\underbrace{T_{\mathbb{C}} f \ldots T_{\mathbb{C}} f}_{p \text { times }}),
$$

where $T_{\mathbb{C}} f$ is the complexification of $T f$ :

$$
T_{\mathbb{C}} f(u)=T f(u)-i T f(J u) .
$$

We observe that $Q_{2}(f)=0$ if and only if $f$ is minimal ( $c f$ Proposition 7.1.3 of [16] and [21],[22]).

We also observe that given a Hitchin representation $\rho$ and a complex structure $J$ on $\Sigma$, on obtain a number $e_{\rho}(J)$, the energy of the associated harmonic mapping. The energy will be the function on Teichmüller space given by $J \mapsto e_{\rho}(J)$.

9.2. Representations, energy and minimal surfaces. However one drawback of this construction is that $H_{J}$ depends on the choice of the complex structure $J$. In particular, it breaks the invariance by the Mapping Class Group and therefore this construction does not give information on the topological nature of $\operatorname{Rep}_{H}\left(\pi_{1}(\Sigma), P S L(n, \mathbb{R})\right) / \mathcal{M}(\Sigma)$. We explain now a more equivariant (with respect to the action of the Mapping Class Group) construction. Let $\mathcal{E}^{(n)}$ be the vector bundle over Teichmüller space whose fibre above the complex structure $J$ is

$$
\mathcal{E}_{J}^{(n)}=\mathcal{Q}(3, J) \oplus \ldots \oplus \mathcal{Q}(n, J) .
$$


We observe that the dimension of the total space of $\mathcal{E}^{(n)}$ is the same as that of $\operatorname{Rep}_{H}\left(\pi_{1}(\Sigma), P S L(n, \mathbb{R})\right)$ since the dimension of the "missing" quadratic differentials in $\mathcal{E}_{J}^{(n)}$ accounts for the dimension of Teichmüller space. account for . We now define the Hitchin map

$$
H\left\{\begin{array}{rl}
: & \rightarrow \mathcal{E}^{(n)} \\
\operatorname{Rep}_{H}\left(\pi_{1}(\Sigma), P S L(n, \mathbb{R})\right. & \mapsto(J, \omega)
\end{array} H_{J}(\omega) .\right.
$$

We are aware that this terminology is awkward since this Hitchin map is some kind of an inverse of what is usually called the Hitchin fibration. From Hitchin construction, it now follows this map is equivariant with respect to the Mapping Class Group action. We quote from [16] the following two results

Theorem 9.2.1. The energy $e_{\rho}$ on Teichmüller space is proper.

Theorem 9.2.2. The Hitchin map is surjective.

Our strategy is to identify $\mathcal{E}^{(n)}$ with the moduli space of equivariant minimal surfaces in the associated symmetric space and to prove that there exists an equivariant minimal surface for every representation by tracking a critical point of the energy. Indeed, harmonic mappings for which the quadratic differential vanishes are conformal, and minimal surfaces are critical points of the energy ([16] and [21],[22]).

Our conjecture in [15] is that the Hitchin map is a homeomorphism, which is also equivalent by the above discussion to the following one

Conjecture 9.2.3. If $\rho$ is a Hitchin representation, then $e_{\rho}$ has a unique critical point.

9.3. The case of $n=3$. Let $\Sigma$ be a locally convex surface in a three affine space $E$ equipped with a volume form. Then we define the Blaschke lift $G$ as a map from $\Sigma$ to the space $\operatorname{Met}(E)$ of euclidean metrics on $E$ of volume 1:

$$
G:\left\{\begin{aligned}
\Sigma & \rightarrow \operatorname{Met}(E) \\
s & \mapsto G(s) \text { such that } G(s)(X, \lambda)=g_{s}(X, X)+\lambda^{2},
\end{aligned}\right.
$$

where $E$ is identified with $T_{s} \Sigma \oplus R$ and $g_{s}$ is the Blaschke metric on $T_{s} \Sigma$.

We know prove the following proposition whose first part is well known.

Proposition 9.3.1. $\Sigma$ is an affine sphere if and only if $G(\Sigma)$ is a minimal surface. Moreover, real part of the cubic holomorphic $q_{3}(G)$ associated to $G$ is related to the Pick invariant $A$ of $\Sigma$ :

$$
12 \cdot g_{s}(A(X) Y, Z)=\Re\left(Q_{3}(G)(X, Y, Z)\right) .
$$

Proof : We just prove the second part of the proposition. We first observe that by definition identifying $T_{g} M$ with the space of symmetric endomorphisms 
of $\mathbb{R}^{3}$. We denote by $X^{*}$ the transpose of $X$,

$$
T G(X)=\Gamma(X)=\left(\begin{array}{cc}
A(X) & X \\
X^{*} & 0
\end{array}\right)
$$

Then

$$
\Gamma(X) \Gamma(Y)=\left(\begin{array}{cc}
A(X) A(Y)+X Y^{*} & A(X) Y \\
X^{*} A(Y) & X^{*} Y
\end{array}\right)
$$

and

$$
\begin{aligned}
\operatorname{trace}(\Gamma(Z) \Gamma(X) \Gamma(Y))= & \operatorname{trace}(A(Z) A(X) A(Y)) \\
& +g(A(Z) X, Y)+g(Z, A(Y) X)+g(Z, A(X), Y)
\end{aligned}
$$

If $\Sigma$ is an affine sphere then, $g(A(Z) X, Y)$ is symmetric in $X, Y, Z$ and

$$
A(Z) J=-J A(Z)
$$

It follows that

$$
\operatorname{trace}(\Gamma(Z) \Gamma(X) \Gamma(Y))=3 \cdot g(A(X) Y, Z) .
$$

Now, using the definition of $T_{\mathbb{C}} G$ we get

$$
\begin{aligned}
\Re\left(Q_{3}(G)(X, Y, Z)\right) & =\Re\left(\operatorname{trace}\left(T_{\mathbb{C}} G(X), T_{\mathbb{C}} G(Y), T_{\mathbb{C}} G(Z)\right)\right) \\
& =\operatorname{trace}(\Gamma(X) \Gamma(Y) \Gamma(Z))-\operatorname{trace}(\Gamma(X) \Gamma(J Y) \Gamma(J Z)) \\
& -\operatorname{trace}(\Gamma(J X) \Gamma(Y) \Gamma(J Z))-\operatorname{trace}(\Gamma(J X) \Gamma(J Y) \Gamma(Z))
\end{aligned}
$$

We also observe that by Equation (19)

$$
g(A(X) J Y, J Z)=-g(A(X) Y, Z) .
$$

Hence

$$
\Re\left(Q_{3}(G)(X, Y, Z)\right)=12 \cdot g(A(X) Y, Z)
$$

Q.E.D.

As an immediate corollary, we obtain

Theorem 9.3.2. For $n=3$, the Hitchin map is a diffeomorphism. Moreover, the energy $e_{\rho}$ has a unique critical point on Teichmüller space which is an absolute minimum.

Indeed, by the previous proposition, the map which associate to a Hitchin representation the Pick invariant of the associated affine sphere and its complex structure is the inverse (up to normalisation by $1 / 12$ ) of the Hitchin map. The second part follows from the fact that a complex structure is a critical point of the energy if and only if the associated harmonic map is minimal.

Therefore, since a Hitchin representation is discrete and torsion free $(c f[15])$, we also have the following corollary 
Corollary 9.3.3. Let $\rho$ be a Hitchin representation of $\pi_{1}(\Sigma)$ in $P S L(3, \mathbb{R})$. Then, there exists a unique minimal surface $\Sigma$ in $\rho\left(\pi_{1}(\Sigma)\right) \backslash S L(3, \mathbb{R}) / S O(3, \mathbb{R})$ such that the injection is a homotopically equivalence.

\section{A HOLOMORPHIC INTERPRETATION}

We finish this paper by another interpretation of Condition (E) 2. We consider the homogeneous space $M=S L(3, \mathbb{R}) / S L(2, \mathbb{R})$, where $S L(2, \mathbb{R})$ is embedded reducibly in $S L(3, \mathbb{R})$. The space $M$ is the space of pairs $(P, u)$ such that $P$ is a plane in $\mathbb{R}^{3}$ and $u$ is a transverse vector to $P$.

We observe that we have the following identification

$$
\begin{aligned}
T_{(P, u)} & =\operatorname{Hom}(P, \mathbb{R} u) \oplus \operatorname{Hom}(\mathbb{R} u, P) \oplus \operatorname{Hom}(\mathbb{R} u, \mathbb{R} u,) \\
& =\underbrace{P^{*} \oplus P}_{W} \oplus \mathbb{R} .
\end{aligned}
$$

We identify $P^{*}$ with $P$ using the 2 -fom $i_{u} \Omega$, where $\Omega$ is the volume form of $\mathbb{R}^{3}$. We equip $W$ with the complex structure

$$
J(u, v)=(-v, u) .
$$

Our last interpretation is the following.

Theorem 10.0.4. Given a Hitchin representation $\rho$ of $\pi_{1}(\Sigma)$ in $P S L(3, \mathbb{R})$, there exists a unique surface $\Sigma$ everywhere tangent to $W$, complex and equivariant under $\rho$.

Proof : Indeed, if $\Sigma$ is equipped with a convex projective structure, we obtain map $i$ from $\Sigma$ to $M$ from the splitting of Theorem 4.2.2. Then, one checks easily that $i(\Sigma)$ is tangent to $W$ and complex. For more details, see Paragraph 2.6 of [12]. Q.E.D.

\section{Appendix A: Geodesics}

We prove the following lemma. Let $M$ be a compact manifold. Let $\nabla$ be a connection on $M$. Let $\gamma$ be a geodesic defined on a maximal interval $I=] a, b[$. Let $g$ be an auxiliary metric. We prove

Lemma 11.0.5. Let $\mu(t)=\sqrt{g(\dot{\gamma}(t), \dot{\gamma}(t))}$. Then, for any $c \in I$

$$
\int_{c}^{b} \mu(s) d s=+\infty \text {. }
$$

Moreover, there exists a constant $K$ such that

$$
\left|\frac{d \mu}{d t}\right| \leq K \mu^{2} .
$$


Proof : We now that $\nabla g$ is bounded since $M$ is compact. It follows that

$$
\left|\frac{d \mu^{2}}{d t}\right|=\left|\nabla_{\dot{\gamma}(t)} g(\dot{\gamma}(t), \dot{\gamma}(t))\right| \leq K \mu^{3} .
$$

The second assertion follows. It follows that if $t>s$ we have

$$
-K(t-s) \leq \frac{1}{\mu(t)}-\frac{1}{\mu(s)} \leq K(t-s) .
$$

We split the end of the proof in two cases

(1) $b=\infty$. Therefore, we have from (23)

$$
\forall t>c, \mu(t) \geq \frac{\mu(c)}{K \mu(c)(t-c)+1} .
$$

Hence

$$
\int_{c}^{\infty} \mu(s) d s \geq \int_{c}^{\infty} \frac{\mu(c}{K \mu(c)(s-c)+1} d s=+\infty .
$$

(2) $b<\infty$. Then, since the geodesic is maximal, it leaves every compact set in the tangent bundle :

$$
\lim _{t \rightarrow b} \mu(t)=\infty .
$$

The Inequality (23) yields

$$
-K(b-s) \leq-\frac{1}{\mu(s)}
$$

Hence

$$
\int_{c}^{b} \mu(s) d s \geq \frac{1}{K} \int_{c}^{b} \frac{1}{b-s} d s=\infty
$$

and the assertion follows.

Q.E.D.

12. Appendix B: Elliptic Monge-Ampère equations.

Let $\Sigma$ be a closed surface. Let $J^{1}(\Sigma, \mathbb{R})$ be the space of 1-jets of functions on $\Sigma$ We denote by $j^{1} f(x)$ the 1 -jet of the function $f$ at $x$. Let

- $G$ be a metric on $\Sigma$ and $\nabla$ its Levi-Civita connexion,

- $d$ be an $\mathbb{R}^{+}$-valued function on $J^{1}(\Sigma, \mathbb{R})$,

- $W$ a mapping from $J^{1}(\Sigma, \mathbb{R})$ to $\operatorname{End}(T \Sigma)$ such that $W\left(j_{1} f(x)\right)$ is a symmetric endomorphism of $T_{x}(\Sigma)$. 
By definition the Monge-Ampère operator associated to $G, d, W$ is

$$
M(f)=d\left(j^{1} f\right) \operatorname{det}\left(\nabla^{2} f+W\left(j^{1} f\right)\right)
$$

where $\nabla^{2} f$ is defined by:

$$
L_{X} \cdot L_{Y} \cdot f-L_{\nabla_{X} Y} \cdot f=G\left(\nabla^{2} f(X), Y\right) .
$$

We will show, using holomorphic curves, the following proposition

Proposition 12.0.6. Let $\left\{g_{n}\right\}_{n \in \mathbb{N}}$ be a sequence of positive functions converging $C^{\infty}$ to a positive function $g_{0}$. Let $\left\{f_{n}\right\}_{n \in \mathbb{N}}$ be a sequence of functions such that

- there exists a constant $K$ such that, for all $n,\left\|f_{n}\right\|_{C^{1}} \leq K$

- $M\left(f_{n}\right)=g_{n}$.

Then after extracting a subsequence, $\left\{f_{n}\right\}_{n \in \mathbb{N}}$ converges $C^{\infty}$ to $f_{0}$ such that

$$
M\left(f_{0}\right)=g_{0} .
$$

We remark that a general theory of Monge-Ampère geometries related to holomorphic curves has been developed in [14].

Proof : We first show that given a positive function $g$, there exists a complex structure $J_{g}$ defined on the contact subbundle $P$ of the tangent bundle of $J^{1}(\Sigma, \mathbb{R})$, such that if for all $f$ such that $M(f)=g$, then the graph $J^{1}(f)$ is holomorphic with respect to $J_{g}$.

We begin by describing the geometry of $J^{1}(\Sigma, \mathbb{R})=T^{*} \Sigma \times \mathbb{R}$. The connexion $\nabla$ gives rise to a decomposition of $T J^{1}(\Sigma, \mathbb{R})$

$$
T J^{1}(\Sigma, \mathbb{R})=T \Sigma \oplus T^{*} \Sigma \oplus \mathbb{R} .
$$

In this decomposition, if we see $j^{1} f$ as a mapping from $\Sigma$ to $J^{1}(\Sigma, \mathbb{R})$, then

$$
T j^{1} f(u)=\left(u, \nabla_{u} d f, d f(u)\right) .
$$

The contact subbundle $P$ at a point $(\omega, \lambda)$ of $J^{1}(\Sigma, \mathbb{R})$ is

$$
P_{(\omega, \lambda)}=\left\{\left(u, \alpha, \omega(u) ; \quad u \in T \Sigma, \quad \alpha \in T^{*} \Sigma\right\} .\right.
$$

Let $i$ be the isomorphism of $T \Sigma$ with its dual coming from the metric $G$. Let $J_{0}$ the complex structure on $T \Sigma$ given by the metric. We identify $P$ with $T \Sigma \oplus T \Sigma$ using the following isomorphism

$$
\psi_{g}:\left\{\begin{aligned}
T \Sigma \oplus T \Sigma & \rightarrow P_{(\omega, \lambda)} \\
(u, v) & \mapsto\left(u, i\left(-\sqrt{g d^{-1}} J_{0} v-W(u)\right), \omega(u)\right)
\end{aligned}\right.
$$

Let $J_{1}$ be the complex structure on $T \Sigma \oplus T \Sigma$ given by : $J_{1}(u, v)=(-v, u)$. Let

$$
J_{g}=\psi_{g} \circ J_{1} \circ \psi_{g}^{-1} .
$$


We now show that $M(f)=g$, if and only if $T\left(j^{1} f\right)(T \Sigma)$ is stable by $J_{g}$. This relies on the following observation: if $B$ is a symmetric positive operator on $T \Sigma$, then

$$
\left(J_{0} B\right)^{2}=-(\operatorname{det} B)^{2} .
$$

In our case, we apply this observation to

$$
B(u)=\sqrt{d g^{-1}}\left(\nabla_{u} \nabla f+W(u)\right) .
$$

Finally, we equip $T \Sigma \oplus T \Sigma$ - and hence $P$ - with the product metric $G_{0}$. We now show :

$$
\operatorname{Area}\left(j^{1} f(\Sigma)\right) \leq A\left(\|f\|_{C^{1}}\right)+C
$$

Indeed

$$
\begin{aligned}
\operatorname{Area}\left(j^{1} f(\Sigma)\right) & =\int_{\Sigma} \operatorname{det}(1+B) \\
& =\operatorname{Area}(\Sigma)+\int_{\Sigma} \operatorname{trace}(B)+\int_{\Sigma} \operatorname{det}(B) .
\end{aligned}
$$

Since $M(f)=g$, we have $\operatorname{det}(B)=1$. Moreover, $B$ being a positive operator

$$
\begin{aligned}
\int_{\Sigma} \operatorname{trace}(B) & \leq \sup \left(\sqrt{d g^{-1}}\right)\left(\int_{\Sigma} \Delta f+\int_{\Sigma} \operatorname{trace}(W)\right) \\
& \leq C\left(\|f\|_{C_{1}}\right)+D
\end{aligned}
$$

where the constants $C$ and $D$ only depend on $g$ and $M$.

We can now translate the hypothesis of our Proposition in an holomorphic language. It follows from our construction that the sequence of graphs $\left\{\Sigma_{n}\right\}_{n \in \mathbb{N}}$ $\left\{j^{1}\left(f_{n}\right)\right\}_{n \in \mathbb{N}}$, where $f_{n}$ satisfies $M\left(f_{n}\right)=g_{n}$, is a sequence of holomorphic curves of bounded area for a sequence of converging complex structures $\left\{J_{g_{n}}\right\}_{n \in \mathbb{N}}$. As it is described in [12], we can apply to this situation Gromov's compactness theorem. Thus the sequence $\left\{\Sigma_{n}\right\}_{n \in \mathbb{N}}$ converges - after taking a subsequence to a holomorphic curve moduli the apparition of bubbles.

For topological reasons, since $\Sigma_{n}$ are graphs over $\Sigma$, the bubbles are subset of the fibres. Therefore, no bubbles can occur since the tangent space of the fibre does not contain any complex subspace. It follows that our sequence of graphs converges smoothly to a graph. Hence that $\left\{f_{n}\right\}$ converges $C^{\infty}$. Q.E.D.

\section{Appendix C: Laplace equations}

Let as before $\Sigma$ be a closed surface. Let $J^{1}(\Sigma, \mathbb{R})$ be the space of 1-jets of functions on $\Sigma$. Let

- $G$ be a metric on $\Sigma$ and $\nabla$ its whose Levi-Civita connexion,

- $F$ be an $\mathbb{R}$-valued function on $J^{1}(\Sigma, \mathbb{R})$ 
The associated Laplace equation is

$$
L_{F}(f)=\Delta f+F\left(j^{1} f\right)=0 .
$$

We are going to sketch - using holomorphic curves - a proof of the following classical result

Proposition 13.0.7. Let $\left\{F_{n}\right\}_{n \in \mathbb{N}}$ be a sequence of functions converging smoothly to $F_{0}$. Let $\left\{f_{n}\right\}_{n \in \mathbb{N}}$ be a sequence of $C^{1}$-bounded functions such that

- $\exists K / \forall n,\left\|f_{n}\right\|_{C^{1}} \leq K$,

- $L_{F_{n}}\left(f_{n}\right)=0$.

Then after extracting a subsequence, $\left\{f_{n}\right\}_{n \in \mathbb{N}}$ converges $C^{\infty}$ to a function $f_{0}$ such that $L_{F_{0}}\left(f_{0}\right)=0$.

Proof : The proof being very similar to the previous one, we are going to be sketchy. In [12], we showed there exists a complex structure $J_{F}$ on the contact subbundle $P$, such that the graph of $j^{1} f$ is holomorphic if and only if $L_{F}(f)=0$.

We now have to obtain a control of the area.

$$
\begin{aligned}
\operatorname{Area}\left(j^{1} f(\Sigma)\right) & \left.\leq \int_{\Sigma} \sqrt{\operatorname{det}\left(1+\left(\nabla^{2} f\right)^{2}\right.}\right) \\
& \leq \int_{\Sigma} 1+\operatorname{trace}\left(\left(\nabla^{2} f\right)^{2}\right)
\end{aligned}
$$

Moreover

$$
\begin{aligned}
\int_{\Sigma} \operatorname{trace}\left(\left(\nabla^{2} f\right)^{2}\right) & =\int_{\Sigma} \sum_{i}\left\langle\nabla_{X_{i}} \nabla f \mid \nabla_{X_{i}} \nabla f\right\rangle \\
& =-\int_{\Sigma} \sum_{i}\left\langle\nabla_{X_{i}} \nabla_{X_{i}} \nabla f \mid \nabla f\right\rangle .
\end{aligned}
$$

It is quite classical to show that

$$
\nabla \Delta f-\sum_{i} \nabla_{X_{i}} \nabla_{X_{i}} \nabla f=H\left(j^{1} f\right)
$$

just depends on the 1-jet of $f$. In particular,

$$
\begin{aligned}
\int_{\Sigma} \operatorname{trace}\left(\left(\nabla^{2} f\right)^{2}\right) & \leq C_{1}(\|f\|)_{C_{1}}-\int_{\Sigma}\langle\nabla \Delta f \mid \nabla f\rangle \\
& \leq C_{2}\left(\|f\|_{C_{1}}\right)+\int(\Delta f)^{2} \\
& \leq C_{3}\|f\|_{C_{1}}+C_{4} .
\end{aligned}
$$

Therefore, as before, $j^{1} f_{n}(\Sigma)$ is a sequence of holomorphic curves of bounded area for a sequence of converging complex structures. Thus, we have convergence up to apparition of bubbles in the fibre. However, in this case the fibre does 
not contain any compact holomorphic curves. We therefore deduce that $\left\{f_{n}\right\}_{n \in \mathbb{N}}$ converges after extracting a subsequence.

\section{Appendix D: the BöChNer Formula}

Proposition 14.0.8. Let $\langle$,$\rangle be a metric of constant curvature -1$ on a manifold $M$ of dimension $n$. Let $\nabla$ be its Levi-Civita connection.Let $\Delta=-\operatorname{trace}\left(\nabla^{2}\right)$ be the Laplacian. Then for any function $\mu$ one has

$$
\Delta\|d \mu\|^{2}=2\langle d(\Delta \mu), d \mu\rangle-2\left\|\nabla^{2} \mu\right\|^{2}+2(n-1)\|d \mu\|^{2} .
$$

Let $R$ be its curvature tensor of $\langle$,$\rangle . If X$ is a vector field and $f$ a function, we use the notation $L_{X} f=d f(X)$. Note that if $\alpha$ is a 1-form, then $L_{X}(\alpha(Y)=$ $\nabla_{X} \alpha(Y)+\alpha\left(\nabla_{X} Y\right)$. Let $x_{0}$ be a point in $M$. Let $\left(e_{1}, \ldots e_{n}\right)$ be an orthornormal frame in the neighbourhood of $x_{0}$ such that $\nabla_{e_{i}} e_{j}=0$ at $x_{0}$. Then at the point $x_{0}$

$$
\Delta f=L_{e_{i}} L_{e_{i}} f
$$

Observe also that at $x_{0}$

$$
\left(\nabla_{e_{i}} \nabla_{e_{j}} d \mu\right)\left(e_{k}\right)=L_{e_{i}}\left(\nabla_{e_{j}} d \mu\left(e_{k}\right)\right)=L_{e_{i}}\left(\nabla_{e_{k}} d \mu\left(e_{j}\right)\right)=\left(\nabla_{e_{i}} \nabla_{e_{k}} d \mu\right)\left(e_{j}\right)
$$

Hence

$$
\begin{aligned}
\Delta\|d \mu\|^{2} & =-\sum_{i} L_{e_{i}} L_{e_{i}}\langle d \mu, d \mu\rangle \\
& =-2 \sum_{i} \sum_{j}\left\langle\nabla_{e_{i}} \nabla_{e_{i}} d \mu, d \mu\right\rangle-2 \sum_{i}\left\langle\nabla_{e_{i}} d \mu, \nabla_{e_{i}} d \mu\right\rangle .
\end{aligned}
$$

Now we remark that at $x_{0}$

$$
\sum_{i}\left\langle\nabla_{e_{i}} d \mu, \nabla_{e_{i}} d \mu\right\rangle=\sum_{j} \sum_{i} \nabla_{e_{i}} d \mu\left(e_{j}\right) . \nabla_{e_{i}} d \mu\left(e_{j}\right)=\left\|\nabla^{2} \mu\right\|^{2} .
$$

Moreover at $x_{0}$,

$$
\begin{aligned}
\sum_{i}\left\langle\nabla_{e_{i}} \nabla_{e_{i}} d \mu \cdot d \mu\right\rangle= & \left.\sum_{j} \sum_{i}\left(\nabla_{e_{i}} \nabla_{e_{i}} d \mu\right)\left(e_{j}\right)\right) d \mu\left(e_{j}\right) \\
= & \sum_{j} \sum_{i}\left(\nabla_{e_{i}} \nabla_{e_{j}} d \mu\right)\left(e_{i}\right) \cdot d \mu\left(e_{j}\right) \\
= & \left.\sum_{j} \sum_{i}\left(R\left(e_{i}, e_{j}\right) d \mu\right)\left(e_{i}\right)\right) \cdot d \mu\left(e_{j}\right) \\
& +\sum_{j} \sum_{i}\left(\nabla_{e_{j}} \nabla_{e_{i}} d \mu\right)\left(e_{i}\right) \cdot d \mu\left(e_{j}\right) \\
= & -\sum_{j} d \mu\left(\sum_{i} R\left(e_{i}, e_{j}\right) e_{i}\right) \cdot d \mu\left(e_{j}\right) \\
& +\sum_{j} L_{e_{j}}\left(\nabla_{e_{i}} d \mu\left(e_{i}\right)\right) \cdot d \mu\left(e_{j}\right) .
\end{aligned}
$$


Finally, we have that

$$
\begin{aligned}
\sum_{i} R\left(e_{i}, e_{j}\right) e_{i} & =(n-1) e_{j}, \\
l \sum_{j} L_{e_{j}}\left(\nabla_{e_{i}} d \mu\left(e_{i}\right)\right) . d \mu\left(e_{j}\right) & =-\sum_{j} L_{e_{j}} \Delta \mu . d \mu\left(e_{j}\right)=-\langle d \Delta \mu, d \mu\rangle .
\end{aligned}
$$

Combining Equations (36), (37), (38), (39) and (40) together, one gets Formula (35). Q.E.D.

\section{REFERENCES}

[1] E. Calabi. Complete affine hyperspheres. Instituto Nazionale di Alta Matematica, Symposia Mathematica 10, 19-39 (1972)

[2] S.-Y. Cheng, S.-T. Yau. On the regularity of the Monge-Ampère equation $\operatorname{det}\left(\left(\partial^{2} u / \partial x^{i} \partial x^{j}\right)\right)=F(x, u)$. C. P. A. M. 30, 41-68 (1977).

[3] S.-Y. Cheng, S.-T. Yau. Complete affine hyperspheres. part I. the completeness of affine metrics. C. P. A. M. 39 (6), 839-866 (1986).

[4] Choi Suhyoung, W. Goldman. Convex real projective structures on closed surfaces are closed Proc. Amer. Math. Soc. 118 (1993), 2, 657-661.

[5] W. Goldman. Convex real projective structures on compact surfaces. J. Differential Geom. 31 (1990) 3, 791-845.

[6] S. Gigena. On a conjecture by Calabi. Geometriae Dedicata, 11 387-396 (1981)

[7] D. Gilbarg, N. Trudinger. Elliptic partial differential equations of second order. Classics in Mathematics. Springer-Verlag, Berlin, 2001.

[8] N. Hitchin. Lie Groups and Teichmüller spaces. Topology 31 (1992), no. 3, 449-473.

[9] K. Corlette. Flat G-bundles with canonical metrics. J. Differential Geom. 28 (1988), no. 3, 361-382.

[10] J.-L. Koszul Variétés localement plates et convexité. Osaka J. Math. 2 (1965) , 285-290.

[11] F. Labourie. Existence d'applications harmoniques tordues à valeurs dans les variétés à courbure négative. Proc. Amer. Math. Soc. 111 (1991), no. 3, 877-882.

[12] F. Labourie. Exemples de courbes pseudo-holomorphes en géométrie riemannienne. in Pseudo-holomorphic curves in symplectic geometry (M. Audin ed.) Progress in Maths (Birkhaüser) 1994

[13] F. Labourie. $\mathbb{R P}^{2}$-structures et differentielles cubiques holomorphes. Proceedings of the GARC Conference in Differential Geometry, Seoul National University, 1997.

[14] F. Labourie. Problèmes de Monge-Ampère, courbes pseudo-holomorphes et laminations. G.A.F.A 7, 496-534 (1997).

[15] F. Labourie. Anosov Flows, Surface Groups and Curves in Projective spaces. Invent. Math. 165 (2006), no. 1, 51-114

[16] F. Labourie. Cross Ratios, Anosov Representations and the Energy Functional on Teichmüller Space. submitted

[17] A.-M. Li. Calabi conjecture on hyperbolic affine hyperspheres. Math. Zeit. 203 483-491 (1990)

[18] A.-M. Li. Calabi conjecture on hyperbolic affine hyperspheres (2). Math. Ann. 293 485-493 (1992)

[19] J. Loftin. Affine spheres and convex $\mathbb{R P}^{n}$-manifolds. Amer. J. Math. 123 (2001), no. 2, $255-274$.

[20] T. Sasaki. Hyperbolic affine hyperspheres Nagoya Math. Journal, 77 107-123 (1980) 
[21] J. Sacks, K. Uhlenbeck. The existence of minimal immersions of 2-spheres. Ann. of Math. (2) 113 (1981), no. 1, 1-24

[22] R. Schoen, Yau Shing Tung. Existence of incompressible minimal surfaces and the topology of three-dimensional manifolds with nonnegative scalar curvature. Ann. of Math. (2) 110 (1979), no. 1, 127-142.

[23] E.B. Vinberg. The theory of convex homogeneous cones. Tranlations Moscow Math. Soc. (1963) 340-403.

[24] Wang Chang Ping. Some examples of complete hyperbolic affine 2-spheres in $\mathbb{R}^{3}$. Global differential geometry and global analysis (Berlin, 1990), 271-280, Lecture Notes in Math., 1481, Springer, Berlin, 1991.

\section{François LABOURIE}

Univ. Paris-Sud, Laboratoire de Mathématiques, Orsay F-91405 Cedex; CNRS, Orsay cedex, F-91405

E-mail: francois.labourie@math.u-psud.fr 